\title{
The BLMP-1 transcription factor promotes oscillatory gene expression to achieve timely molting
}

${ }^{1}$ Friedrich Miescher Institute for Biomedical Research (FMI), Maulbeerstrasse 66, CH-4058 Basel.

${ }^{2}$ University of Basel, Petersplatz 1, CH-4001 Basel.

${ }^{3}$ Swiss Institute of Bioinformatics, Basel, Switzerland.

${ }^{4}$ Correspondence to: helge.grosshans @ fmi.ch

\begin{abstract}
Gene expression oscillators can coordinate developmental events in space and time. In $C$. elegans, a gene expression oscillator directs rhythmic accumulation of $\sim 25 \%$ of the transcriptome, and thus thousands of transcripts, presumably to control molting, a process of rhythmic skin regeneration. The mechanism and organization of the oscillator are not known. Here, we report that rhythmic RNA polymerase II recruitment to promoters produces transcript level oscillations. We identify BLMP-1, orthologous to the mammalian transcription repressor PRDM1, as a rhythmically accumulating transcription factor that is required for timely molting, and oscillatory gene expression. We propose a dual function for BLMP-1 in shaping oscillatory gene expression and coupling it to a set of direct targets, which ensures cuticular integrity. With mammalian PRDM1/BLIMP1 promoting regular cycles of postnatal hair follicle regeneration, our findings point to the possible existence of a fundamentally conserved clock mechanism in control of rhythmic skin regeneration.
\end{abstract}




\section{Introduction}

Biological 'clocks', based on genetic oscillators, drive dynamic processes ranging from circadian rhythms (Panda et al., 2002) to vertebrate somitogenesis (Aulehla and Herrmann, 2004; Oates et al., 2012). Yet, in many other instances of cyclical developmental processes, the underlying clocks have remained elusive. For instance, although the regenerative cycles of mammalian postnatal hair follicle growth, regression and quiescence, and the nematode molting cycle are both considered to be controlled by molecular clocks, components and mechanisms have remained elusive (Paus and Foitzik, 2004, Monsalve and Frand, 2012).

Nematode molting is a process of skin barrier renewal. It involves the synthesis of a new cuticle from the single-layer epidermis during a period of behavioral quiescence, termed lethargus, and the subsequent shedding of the old cuticle (ecdysis), which demarcates the end of a larval stage (Lažetić and Fay, 2017). Recently, we and others found that in C. elegans, $\sim 25 \%$ of the transcriptome (corresponding to $\sim 3,700$ genes, henceforth referred to as oscillating genes) exhibits high-amplitude oscillations coupled to the molting cycle but with a diversity of peak phases (Hendriks et al., 2014; Kim et al., 2013, Meeuse et al., 2020; reviewed in Tsiairis and Großhans, 2020). Most of the $\sim 3,700$ oscillating genes are expected to constitute oscillator outputs, which allow the oscillator to perform specific physiological functions in molting and possibly other developmental processes (Tsiairis and Großhans, 2020). Thus, they are a manifestation of oscillator activity, rather than components or modulators of oscillator mechanisms, which remain to be identified.

Although rhythmic transcription would provide a parsimonious explanation of mRNA level oscillations, rhythmic circadian mRNA accumulation appears to rely chiefly on co- and post-transcriptional mechanisms, including rhythmic splicing (Koike et al., 2012; Menet et al., 2012; Preußner et al., 2017). Here, we demonstrate that transcript level oscillations are a result 
of rhythmic RNA polymerase II (RNAPII) recruitment to promoters. We identify BLMP-1, an orthologue of the lineage-specifying mammalian transcription factor BLIMP1/PRDM1, as required for timely and robust molting. In C. elegans, BLMP-1 was previously shown to regulate gonad migration and epidermal cell terminal differentiation (Horn et al., 2014; Huang et al., 2014). We now show that BLMP-1 protein accumulates rhythmically and functions repetitively during larval development. BLMP-1 depletion causes an extension of larval molts, with more severely affected animals also exhibiting an extended intermolt and, frequently, death. Loss of BLMP-1 broadly perturbs oscillatory gene expression, including for a subset of genes that it binds and, in a gene-specific manner, activates or inactivates. This compromises cuticular integrity, causing a loss of skin barrier function. Collectively, our data indicate that BLMP-1 contributes to robust oscillator function, relays its activity directly to a set of rhythmically expressed genes, and thereby promotes faithful developmental progression and timely molting. As mammalian BLIMP1/PRDM1 was shown to be important for hair follicle cycles (Telerman et al., 2017), our data suggest a potential function in the currently elusive hair follicle clock (Paus and Foitzik, 2004).

\section{Results}

Rhythmic transcription of oscillating genes is driven by rhythmic RNAPII occupancy

Previous observations that intronic RNA encoded by oscillating genes also oscillates (Hendriks et al., 2014) provided circumstantial evidence for a model of rhythmic oscillating gene transcription. However, technical limitations restricted this analysis to a set of highly expressed genes with long introns, and genuine pre-mRNAs could not be distinguished from excised introns. Hence, we employed temporally resolved DNA-dependent RNA polymerase II (RNAPII) chromatin immunoprecipitation coupled to sequencing (ChIP-seq) to examine 
the dynamics of RNAPII binding to oscillating gene promoters. We used synchronized wildtype worms collected hourly at 22 hours until 33 hours of post-embryonic development at $25^{\circ} \mathrm{C}$ and quantified a 1-kb window around the transcription start sites (TSSs) as a proxy for temporal RNAPII promoter occupancy on annotated oscillating genes (Meeuse et al., 2020). This revealed wide-spread rhythmic binding of RNAPII at the promoters of genes for which we also observed mRNA level oscillations in RNA sequencing data generated from the same samples (Fig 1A, B, Fig S1).

We also detected instances where oscillating mRNA levels were not accompanied by rhythmic RNAPII promoter binding. It is possible that this might reflect instances of posttranscriptional regulation. However, we consider this more likely a technical limitation, because we noticed a general reduction of amplitudes in the ChIP-sequencing relative to the RNA-sequencing experiment, probably reflecting a reduced dynamic range of the former over the latter (see also below). This limitation notwithstanding, the data support the notion that rhythmic transcription is a major contributor to rhythmic transcript accumulation and 15 specifically point to rhythmic recruitment of RNAPII as a relevant mechanism.

Promoter-driven gfp reporter transgenes recapitulate transcription of endogenous genes

To understand in more detail how rhythmic transcription drives mRNA level oscillations, we characterized transcriptional reporters that contained the putative promoters (either $2 \mathrm{~kb}$ upstream of the ATG or until the next upstream gene) of oscillating genes fused to a sequence for encoding destabilized, nucleus-localized green fluorescent protein (GFP). We chose promoters from genes with a variety of peak expression phases. To exclude 3'UTR-mediated posttranscriptional regulation, the reporters further contained the unc-54 3'UTR, which we selected because unc-54 did not display transcript level oscillation in our mRNA sequencing 
time courses (Hendriks et al., 2014; Meeuse et al., 2020) and because its 3'UTR appears devoid of regulatory activity (Brancati and Großhans, 2018). All reporters were integrated into the $C$. elegans genome in single copy at the same defined genomic locus.

To assess the extent to which transgenes, and thus promoter activity, could recapitulate endogenous rhythmic gene activity, we compared dynamic changes of abundance of the endogenous transcript and its $g f p$ mRNA counterpart within the same worm lysates obtained by sampling synchronized worm populations hourly between $22-37$ hours after plating starved L1 larvae on food (Fig 1C). In each of 8 cases that we examined, chosen to represent a variety of peak phases and amplitudes, we observed rhythmic reporter transcript accumulation. Remarkably, the patterns of the endogenous transcripts and their derived reporters were also quantitatively highly similar, i.e., in all tested cases except one, peak phases and amplitudes were comparable (Fig 1C, Fig S2A). (We suspect, but have not examined further, that in the one case where we observe a deviation, R12.E2.7p in Fig S2A, the reporter may lack relevant promoter or intronic enhancer elements.) Furthermore, in the case of $F 58 H 1.2$, the reporter RT-qPCR time course recapitulated high-amplitude oscillation despite a much more modest oscillatory signal in the ChIP-seq experiment (Fig S2B), further supporting the notion that the differences in amplitudes between ChIP-seq and mRNA-seq probably are of technical nature and do not primarily arise from post-transcriptional regulation of the transcripts. are sufficient to recapitulate endogenous transcript dynamics. 
Rhythmic transcription and RNAPII recruitment suggested an involvement of rhythmic transcription factor activity in generating transcript level oscillations. To identify such factors, we exploited the coupling between mRNA oscillations and the molting cycle (Meeuse et al., 2020) and used a targeted RNAi-approach to identify transcription factors required for timely molting (Meeuse et al., in preparation). We found that depletion of BLMP-1 caused extended molt durations.

To characterize this phenotype further, we examined a larger number of animals using a luminescence-based assay that facilitates detection of molt entry and molt exit, and thus determination of molt, intermolt and larval stage duration, on single animals in high throughput (Meeuse et al., 2020; Olmedo et al., 2015). Briefly, animals expressing a luciferase transgene constitutively were cultured in a multi-well plate in a temperature-controlled luminometer in the presence of food and luciferin. Continuous light emission results, except during lethargus (molt), when animals do not feed so that luciferin uptake does not occur. Consistent with the screen results, blmp-1(RNAi) caused a lengthening of molts relative to mock-depleted animals (Fig 2A, Fig S3A,E).

Surprisingly, we observed in some but not all experiments largely unchanged larval stage durations, because lengthening of molts was compensated by a shortening of intermolts (Fig 2A-C, Fig S3A-C, E-G). We wondered whether the variable effects on intermolt duration reflected differences in depletion achieved in different RNAi experiments. To test this, we tagged the endogenous protein with an auxin-inducible degron (AID) in animals expressing Arabidopsis TIR1 ubiquitously (Zhang et al., 2015) to achieve a better control over degradation. Following addition of different amounts of auxin to embryos to trigger AIDBLMP-1 degradation, we indeed found an auxin dose-dependent effect: Increasing concentrations of auxins caused increasing molt durations, up to a maximum of a 2-fold increase at $1 \mathrm{mM}$ auxin (Fig 2D). At the same time, intermolts decreased at lower auxin 
concentrations but increased at high auxin concentrations (Fig 2E), thereby resulting in a largely constant larval stage duration at low, but an increased duration at high auxin concentration (Fig 2F).

Finally, when we examined blmp-1(tm548) mutant animals, considered null mutant (Horn et al., 2014; Huang et al., 2014), many animals arrested larval development or died (Fig $2 \mathrm{G})$, as inferred from a gradual loss of luminescence after exit from the first molt (Fig S3MO). For the subset of blmp-1(tm548) animals that developed through all larval stages, all four molts were greatly extended relative to wild-type animals (Fig $2 \mathrm{H}$ ), whereas intermolts were more variably affected, with a substantial lengthening detectable for intermolt 2 (I2), but much less so for I1 and I3, and in fact a decrease for I4 (Fig 2I). From M1 onwards, molt, intermolt and larval stage durations also became more variable across individuals (Fig 2H-J).

We conclude that BLMP-1 is important for normal progression of development with molting being particularly sensitive to reduction in BLMP-1 levels.

Whereas luminescence is low in wild-type animals during lethargus, when luciferin is not ingested, we consistently observed elevated luminescence during M2 through M4 in both blmp-1 RNAi-treated and blmp-1(tm548) mutant animals (Fig 3A, B, Fig S3D, H, L). To examine whether this could reflect luciferin uptake via routes other than ingestion; i.e., by penetration of the cuticular barrier, we examined cuticular permeability to the DNA stain Hoechst 33258 (Moribe et al., 2004). We incubated synchronized L4 stage larvae with the dye and quantified the fraction of animals with nuclear DNA staining. While such staining was rarely observed in wild-type animals, it was highly penetrant in blmp-1 mutant animals, comparable to the known cuticle defective strain bus-8(e2885), which we used as a positive 
Depletion of BLMP-1 from L1 stage on affected the durations of all larval stages (Fig 2, S3). To investigate whether this reflected a recurring function of BLMP-1 for each molt, we utilized the aid::blmp- 1 strain to achieve temporally controlled depletion of BLMP-1. When we added auxin ( $250 \mu \mathrm{M}$ final concentration) to animals at various times during the L2 stage, we observed an extended molt 3 in all animals (Fig 4A, B). Moreover, animals that had experienced BLMP-1 depletion sufficiently early in L2 also exhibited an extended molt 2, with the size of the effect depending on the time of application. We observed analogous results, now for molt 3 and molt 4, when we applied auxin at different times during the L3 stage (Fig S4). during development (Fig 4C; Hendriks et al., 2014; Meeuse et al., 2020). Hence, we wondered whether BLMP-1 protein accumulated rhythmically. Since previous studies that had examined BLMP-1 protein accumulation either lacked sufficient temporal resolution to detect dynamics within a given stage (Hyun et al., 2016) or did not examine endogenous protein (Horn et al., 2014; Stec et al., 2021), we engineered a strain to express 3xflag::blmp-1 from the endogenous locus and examined BLMP-1 levels by Western Blotting at high temporal resolution. Sampling over extended periods of time during larval development at $20^{\circ} \mathrm{C}$ or $25^{\circ} \mathrm{C}$ revealed a dynamic pattern of BLMP-1 accumulation (Fig 4D,E). Specifically, using a 
parallel luciferase assay to approximate time of molting (Methods), we observed low levels of BLMP-1 protein during the molt and high levels during the intermolt.

Taken together, these data suggest that rhythmic BLMP-1 accumulation during development underlie its rhythmic activity during molting.

BLMP-1 controls the expression of a large set of molting genes

To understand the molecular function of BLMP-1, we examined a publicly available BLMP1 ChIPseq dataset (GSE25803, modENCODE_DCC: 2612) to identify the genes that it binds. From two independent replicates, we identified 856 genes bound by BLMP-1 in their promoters (defined as a 1,000bp region centered around the ATG start codon) (Fig S5). Since transcription factor binding tends to be poorly predictive of transcriptional regulation (Cusanovich et al., 2014), we further performed two replicate RNA sequencing time-courses on synchronized populations of blmp-1(tm548) mutant and wild-type animals grown at $25^{\circ} \mathrm{C}$. We plated synchronized early L1 stage larvae and sampled hourly over $24 \mathrm{~h}$, thus covering the first intermolt, during which blmp-1 mutant animals develop synchronously and at largely normal rate, the greatly extended, yet still relatively synchronous molt 1 , and an extended and variable intermolt 2, up to and into molt $2($ Fig $2 \mathrm{H}, \mathrm{I})$. Unlike in liquid medium in the luciferase assay, viability of blmp-1 mutant animals appears largely unimpaired when grown on plate, as also observed by others (Stec et al., 2021).

As changes in developmental tempo would cause apparent changes in gene expression patterns that are not a direct consequence of BLMP-1 dysfunction (Fig S6A, B, Tsiairis and Großhans, 2020), we temporally realigned the gene expression of all time-courses to the replicate 2 of the blmp-1(tm548) mutant time course (Fig S6, see Methods). A dynamic threshold considering the magnitude as well as the significance of the effect (Fig. S7A, 
Methods $)$ then allowed us to identify among all expressed genes $(n=16,720)$ those that were differentially expressed between $\operatorname{blmp}-1(\operatorname{tm} 548)$ and wild-type $(\mathrm{n}=1,655$, Fig 5A).

We confirmed the validity of the realignment approach through an orthogonal, single worm time-lapse imaging approach (Meeuse et al., 2020), which allowed us to observe larval stage durations, and thus developmental tempo, directly and in parallel to the expression of a given transcriptional $g f p$ reporter. We selected the promoters of two genes, F11E6.3 and F16B4.4, that were both bound by BLMP-1 (Fig 5C). However, consistent with the notion that transcription factor binding is not predictive of function, endogenous transcript levels of F11E6.3 do not change significantly in the rescaled RNA-seq data (Fig 5B, Fig S6E), and this observation was replicated, and thus validated, by the transcriptional reporter: Expression dynamics were comparable for the F11E6.3p::gfp reporter in BLMP-1 depleted and mock control animals (Fig 5D, Fig S7B - G).

By contrast, BLMP-1 depletion affects both accumulation of the endogenous F16B4.4 transcript and expression of a F16B4.4p reporter (Fig 5D, Fig S7B - G). Indeed, notably, both the shape and peak time of the $F 16 B 4.4 p$ reporter expression changed substantially upon BLMP-1 depletion, validating the role of BLMP-1 in generating oscillatory gene expression patterns.

Intersection of the BLMP-1 ChIP-seq and RNA-seq data sets enabled us to identify 248 genes as high-confidence BLMP-1 targets, based on the fact that BLMP-1 both bound their promoters and affected their expression (Fig 6A). Spatial expression patterns further corroborated their designation as direct BLMP-1 targets: Single cell expression data from wild-type animals (Cao et al., 2017) revealed that these genes were preferentially expressed in tissues with high blmp-1 levels, in particular seam cells, non-seam hypodermis, vulval precursor cells, excretory cells, socket cells and rectum (Fig 6B, Fig S8). By contrast, little 
spatial bias occurred for genes that were not significantly changed in the blmp-1(tm548) RNA sequencing time course; a similar fraction $(\sim 10 \%)$ was detected in each tissue, without positive correlation to blmp-1 expression levels (Fig 6C, Fig S8). We note that distal tip cells (DTCs) of the somatic gonad exhibited high blmp-1 RNA levels but little or no evidence for an enrichment of the direct BLMP-1 targets identified by us, likely reflecting a limitation of bulk ChIP-seq and RNA-seq assays to detect such effects in a tissue comprising only 2 cells per animal.

We performed gene ontology (GO) analysis for differentially expressed genes, encompassing both direct targets and secondarily affected genes, as well as for direct targets only. We found that both sets exhibited a strong and significant enrichment of cuticle- and molting-related terms (Fig 6D), providing a molecular explanation of the molting defects manifest in blmp-1 mutant animals.

\section{BLMP-1 promotes oscillatory gene expression}

We noticed an abundance of rhythmically expressed genes among both the genes that were differentially expressed in blmp-1 mutant animals (Fig. 5A), and the direct BLMP-1 targets (Fig 7A). Indeed, quantification revealed a strong enrichment of oscillating genes in all BLMP-1-related categories, i.e., BLMP-1-bound genes, differentially expressed genes, direct BLMP-1 targets relative to all unchanged genes (Fig. 7B). This effect was particularly striking among direct BLMP-1 targets, of which a staggering $88.7 \%$ were oscillating genes. Hence, regulation of oscillating gene expression is the predominant activity of this transcription factor.

To understand if and how BLMP-1 shaped gene expression oscillations, we examined the peak phases of its direct targets in wild-type animals. We observed that, in wild-type 
animals, these genes had a strong preference for peak expression in interphase (Fig 7C, D), when BLMP-1 protein levels are also high (Fig. 4E). This peak phase preference was also detectable, albeit at reduced magnitude, when examining only BLMP-1 binding, without functional validation (Fig 7E). By contrast, when we looked at all differentially expressed genes (i.e., direct targets and secondary targets, which lack BLMP-1 promoter binding) or only at secondary target genes, this peak phase bias was greatly reduced (Fig 7F,G). Thus, BLMP-1 appears to have two, presumably connected, functions: It promotes oscillatory expression of its direct targets by modulating their transcription rhythmically. In addition, it promotes oscillatory expression of a larger set of genes that lack a clear peak phase signature and which it does not bind directly. These secondary targets thus appear to depend on promotion of core oscillator activity through BLMP-1. Consistent with this notion, oscillatory expression of secondary targets was dramatically impaired in blmp-1 mutant animals (Fig. S9).

To explore mechanisms of direct target regulation, we used hierarchical clustering of the fold changes to resolve six groups of genes with distinct behaviors (Fig 7A,H). As for the secondary targets, a general impairment in oscillatory expression was evident, frequently manifesting in a reduction in oscillation amplitude in blmp-1(tm548) mutant animals (Fig $7 \mathrm{H})$. Yet, the patterns differed for distinct clusters. Thus, in clusters 1 and 6 , trough expression levels were up while peak levels remained unaffected. By contrast, in cluster 3 peak levels were decreased. (More complicated patterns in the other clusters may reflect a greater diversity in gene peak expression phases, diluting observable differences.) These findings indicate that BLMP-1 may act as both a transcriptional repressor and a transcriptional activator, depending on the identity of its target. 


\section{Discussion}

The biological functions and mechanism of the recently discovered C. elegans gene expression oscillator have thus far remained poorly defined (Hendriks et al., 2014; Kim et al., 2013; Meeuse et al., 2020). Here, we have identified rhythmic transcription as a major driver of oscillatory mRNA accumulation, and uncovered and characterized a transcription factor, BLMP-1, that promotes oscillatory gene expression and timely molting. Both blmp-1 mRNA and protein levels oscillate, and BLMP-1 is needed repetitively during larval development, for each molt. BLMP-1 promotes rhythmic gene expression by both activating and repressing transcription of direct target genes. We propose that BLMP-1 functions as a potential core oscillator gene that additionally relays oscillator activity to a subset of output genes. These functions serve to achieve timely, well-coordinated molts and, in consequence, a functional cuticle.

\section{Rhythmic transcription generates oscillating mRNA levels}

The previous demonstration of rhythmic intronic RNA accumulation (Hendriks et al., 2014) implicated rhythmic transcription in the $C$. elegans larval oscillator. Time-resolved RNA pol II ChIP sequencing and reporter gene assays presented here now provide direct experimental support of this idea: Globally, ChIP-seq and mRNA-seq experiments yielded highly similar patterns, and several promoter fusion transgenes recapitulated the rhythmic accumulation of endogenous transcripts both qualitatively, as also noted in other instances (Frand et al., 2005; Hao et al., 2006), and quantitatively.

Although our results demonstrate a major function of rhythmic RNA polymerase II recruitment in oscillating mRNA accumulation, we emphasize that, at least in specific cases, post-transcriptional regulatory mechanisms may serve to generate, shape or damp 
blmp- 1 is one of $\sim 100$ oscillating genes encoding transcription factors (Hendriks et al., 2014; Meeuse et al., 2020). Although rhythmic mRNA accumulation is not a requirement for rhythmic transcription factor activity (since post-transcriptional mechanisms acting for instance on protein stability or subcellular localization would constitute viable alternatives), it would provide a facile mechanism. Indeed, we find that endogenous BLMP-1 accumulates rhythmically, with notably low levels during molts. Both BLMP-1 mRNA and protein level show greatly reduced dynamics and expression levels after early L4, consistent with the system's transition to a non-oscillatory state (Meeuse et al., 2020). This may explain a previous report of constitutive blmp-1::gfp expression from multicopy transgenes during the L4 stage (Stec et al., 2021). Given that BLMP-1 is an established target of the DRE-1 E3 ubiquitin ligase (Horn et al., 2014; Huang et al., 2014), it will be interesting to determine in future work whether and to which extent DRE-1 modulates BLMP-1's rhythmic accumulation and activity.

Close coupling between molting and oscillatory gene expression (Meeuse et al., 2020) renders progression of molting a potential proxy for oscillator activity in screens. Our characterization of BLMP-1, identified by a "long and variable molt" phenotype that resulted from RNAi-mediated BLMP-1 depletion, supports this notion. Conversely, this finding 
provides strong evidence to support a function of the gene expression oscillator as a molting clock.

BLMP-1's effect on developmental progression is dose-dependent, such that molt durations became extended in an auxin dose-dependent manner when we used the aid system to deplete BLMP-1. For intermolts, by contrast, a non-monotonic relationship occurs: At low auxin levels, intermolt duration is reduced, compensating for the extension of molting period duration and thereby resulting in unaltered larval stage duration. However, beyond a certain auxin level, intermolt durations begin to increase again, to the point that they exceed durations observed in wild-type animals, and resulting in an increase of larval stage durations. This was also evident in blmp-1 null mutant animals. Whether increased intermolt durations reflect a second function of BLMP-1 that is less sensitive to depletion than molts or constitute an indirect consequence of particularly large increases of molt durations, remains to be established.

BLMP-1 exhibits activating and repressing functions on direct targets to promote their oscillatory expression

When animals are grown on solid food plates, BLMP-1 appears dispensable for viability (this report, (Stec et al., 2021), suggesting some redundancy in the molting clock that can maintain sufficient functionality for survival. However, under other, presumably more stressful, growth conditions, i.e., in microchambers, microfluidics devices (Stec et al., 2021) or in multiwell plates in liquid media, lack of BLMP-1 greatly impairs viability of blmp-1 null mutant animals, an effect potentially connected to their defective cuticle. Even under the conditions that allow survival of blmp-1 null mutant animals, its absence causes substantial transcriptional dysregulation preferentially affecting oscillating genes. Among the six clusters 
of direct BLMP-1 targets, i.e., genes that are dysregulated by blmp- 1 loss and normally bound by BLMP-1 in their promoters, we commonly observed reduced oscillation amplitudes.

However, depending on the cluster, different effects contributed to this result: in some cases, peak expression levels decreased, whereas in others, trough levels increased. We emphasize 5 that BLMP-1 binds to the promoters of all these genes, suggesting direct effects. Indeed, although Blimp1/PRDM1 proteins are well established transcriptional repressors (John and Garrett-Sinha, 2009), others recently reported a role of C. elegans BLMP-1 and mouse Blimp1 in transcriptional activation (Minnich et al., 2016; Stec et al., 2021). Our data add support the notion that Blimp1/Prdm1 proteins may generally be capable of both repressing and activating target genes.

\section{BLMP-1 as a core clock gene in the C. elegans developmental oscillator}

Recently, Stec et al. (2021) reported that BLMP-1 functioned as a pioneer transcription factor and suggested that it provide a permissive activity for dynamic gene expression. For instance, BLMP-1 binding would open up chromatin at target loci to prime them for access by rhythmically active, instructive transcription factors. This model would accommodate the authors' observation of invariant BLMP-1::GFP level produced from a multicopy array during the L4 stage and of a lack of overt peak phase preference, by visual inspection, among the oscillating genes that BLMP-1 bound. However, as discussed above, endogenous blmp-1 mRNA and protein both accumulate rhythmically, and BLMP-1 is repetitively required for molting, in each larval stage. Moreover, our quantitative analysis of the direct, functional targets of BLMP-1, i.e., the genes that are both bound and regulated by BLMP-1, revealed a clear peak phase preference, and thus a molecular signature of rhythmic BLMP-1 activity. Hence, our data support a model where this rhythmic BLMP-1 activity endows BLMP-1 with 
an instructive role in setting temporal gene expression and molting patterns. Incidentally, although a quantitative analysis does reveal a peak phase preference in BLMP-1 bound target genes, this effect is indeed much more pronounced among direct targets (Fig. 7D, E) reaffirming the importance of assaying transcription factor activity directly rather than relying on binding events for proxy (Cusanovich et al., 2014).

Is BLMP-1 a core clock gene? A definitive answer will require tools to manipulate BLMP-1 temporal activity patterns more systematically and/or elucidation of its regulatory interactions with other core clock genes. However, in the circadian clock field, a set of characteristics that include arrhythmicity and strong period increase (or decrease) of the circadian rhythm are considered strong indicators for core clock components (reviewed in Takahashi 2004). By this measure, we would argue for a qualified yes, as blmp-1 loss greatly increases molting cycle periods and their variability, and impairs oscillatory gene expression. Notably, oscillatory expression of numerous secondary targets is compromised, and a lack of a peak phase preference among these genes supports a core oscillator defect. Yet, similar to certain core circadian clock genes such as fly CYC/CLK (Patke et al., 2020), BLMP-1 additionally appears to relay directly core oscillator activity to a subset of output genes, as evidenced by its binding and regulation of $>200$ oscillating genes in specific expression phases. Potentially, this provides the molecular underpinning for its more prominent effect on molts instead of intermolts and the major cuticle defects that result from BLMP-1 depletion.

\section{Blimp1/Prdm1 functions in temporal hair follicle patterning}

Mammalian hair follicles undergo regular cycles of expansion (anagen), regression (ketagen) and quiescence (telogen). While it has long been assumed that this cyclical process of skin regeneration is driven by a molecular clock (Paus and Foitzik, 2004), its mechanism and 
molecular nature has remained enigmatic. However, Telerman et al. (2017) recently showed that mouse Blimpl/Prdml was dynamically expressed in the dermal papilla compartment of the hair follicle, with high levels specifically observed during anagen induction and through advanced anagen. Moreover, dermis-specific Blimpl deletion resulted in a delayed onset, and thereby duration, specifically of anagen. Finally, mirroring the cuticular defects seen in blmp1 mutant $C$. elegans, prdm 1 mutant mice exhibit skin barrier defects. Given these striking parallels, we propose that $C$. elegans is a suitable model to study general principles of skin regeneration.

Molting is frequently defined as a process of exoskeleton replacement in ecdysozoa (Lažetić and Fay, 2017) that is unique to this group of animals (Ewer, 2005), constituting one of their defining features (Valentine and Collins, 2000). Yet, rhythmic renewal of the integument (outer shell) is seen widely also in vertebrate, as illustrated by periodic skin shedding in reptiles and amphibians, feather shedding in birds, or hair shedding in mammals such cats or rabbits, and it may indeed be universal among animals (Stenn and Paus, 2001). Our results suggest the possibility of a common basis of vertebrate and invertebrate molting processes and specifically imply that homeostatic skin regeneration may involve similar mechanisms across the animal kingdom. Specifically, we propose that studying the hair follicle from the point of view of a gene expression oscillator could prove highly productive as a means to understand the hair follicle clock.

\section{References}

Anders, S., Pyl, P.T., and Huber, W. (2015). HTSeq-A Python framework to work with highthroughput sequencing data. Bioinformatics 31, 166-169.

Arribere, J.A., Bell, R.T., Fu, B.X.H., Artiles, K.L., Hartman, P.S., and Fire, A.Z. (2014). Efficient marker-free recovery of custom genetic modifications with CRISPR/Cas9 in caenorhabditis elegans. Genetics 198, 837-846.

Aulehla, A., and Herrmann, B.G. (2004). Segmentation in vertebrates: Clock and gradient finally joined. Genes and Development 18, 2060-2067. 
Bethke, A., Fielenbach, N., Wang, Z., Mangelsdorf, D.J., and Adam, A. (2009). Nuclear hormone receptor regulation of microRNAs controls developmental progression. Science 324, 95-98.

Bolstad, B. (2001). Probe Level Quantile Normalization of High Density Oligonucleotide Array Data.

Bolstad, B. (2021). preprocessCore: A collection of pre-processing functions. /preprocessCore.

Bolstad, B.M., Irizarry, R.A., Åstrand, M., and Speed, T.P. (2003). A comparison of normalization methods for high density oligonucleotide array data based on variance and bias. Bioinformatics 19 , 185-193.

Brancati, G., and Großhans, H. (2018). An interplay of miRNA abundance and target site architecture determines miRNA activity and specificity. Nucleic Acids Research 46, 3259-3269.

Cao, J., Packer, J.S., Ramani, V., Cusanovich, D.A., Huynh, C., Daza, R., Qiu, X., Lee, C., Furlan, S.N., Steemers, F.J., et al. (2017). Comprehensive single-cell transcriptional profiling of a multicellular organism. Science 357, 661-667.

Cusanovich, D.A., Pavlovic, B., Pritchard, J.K., and Gilad, Y. (2014). The Functional Consequences of Variation in Transcription Factor Binding. PLoS Genetics 10, e1004226.

Dobin, A., Davis, C.A., Schlesinger, F., Drenkow, J., Zaleski, C., Jha, S., Batut, P., Chaisson, M., and Gingeras, T.R. (2013). STAR: Ultrafast universal RNA-seq aligner. Bioinformatics 29, 15-21.

Edgar, R. (2002). Gene Expression Omnibus: NCBI gene expression and hybridization array data repository. Nucleic Acids Research 30, 207-210.

Ewer, J. (2005). How the ecdysozoan changed its coat. PLoS Biology 3, 1696-1699.

Frand, A.R., Russel, S., and Ruvkun, G. (2005). Functional genomic analysis of c. elegans molting. PLoS Biology 3.

Fraser, A.G., Kamath, R.S., Zipperlen, P., Martinez-Campos, M., Sohrmann, M., and Ahringer, J. (2000). Functional genomic analysis of C. elegans chromosome I by systematic RNA interference. Nature 408, 325-330.

Frøkjær-Jensen, C., Davis, M.W., Ailion, M., and Jorgensen, E.M. (2012). Improved Mos1-mediated transgenesis in C. elegans. Nature Methods 9, 117-118.

Gaidatzis, D., Lerch, A., Hahne, F., and Stadler, M.B. (2015). QuasR: Quantification and annotation of short reads in R. Bioinformatics 31, 1130-1132.

Gibson, D.G., Young, L., Chuang, R.-Y., Venter, J.C., Hutchison, C.A., and Smith, H.O. (2009). Enzymatic assembly of DNA molecules up to several hundred kilobases. Nature Methods 6, 343-345.

Hao, L., Johnsen, R., Lauter, G., Baillie, D., and Bürglin, T.R. (2006). Comprehensive analysis of gene expression patterns of hedgehog-related genes. BMC Genomics 7, 280.

Hendriks, G.J., Gaidatzis, D., Aeschimann, F., and Großhans, H. (2014). Extensive Oscillatory Gene Expression during C.elegans Larval Development. Molecular Cell 53, 380-392.

Horn, M., Geisen, C., Cermak, L., Becker, B., Nakamura, S., Klein, C., Pagano, M., and Antebi, A. (2014). DRE-1/FBXO11-dependent degradation of BLMP-1/BLIMP-1 governs C. elegans developmental timing and maturation. Developmental Cell 28, 697-710.

Huang, T.F., Cho, C.Y., Cheng, Y.T., Huang, J.W., Wu, Y.Z., Yeh, A.Y.C., Nishiwaki, K., Chang, S.C., and Wu, Y.C. (2014). BLMP-1/Blimp-1 Regulates the Spatiotemporal Cell Migration Pattern in C. elegans. PLoS Genetics 10. 
Hyun, M., Kim, J., Dumur, C., Schroeder, F.C., and You, Y.J. (2016). BLIMP-1/BLMP-1 and metastasis-associated protein regulate stress resistant development in Caenorhabditis elegans. Genetics 203, 1721-1732.

John, S.A., and Garrett-Sinha, L.A. (2009). Blimp1: A conserved transcriptional repressor critical for differentiation of many tissues. Experimental Cell Research 315, 1077-1084.

Kamath, R.S., and Ahringer, J. (2003). Genome-wide RNAi screening in Caenorhabditis elegans. Methods 30, 313-321.

Katic, I., Xu, L., and Ciosk, R. (2015). CRISPR/Cas9 genome editing in Caenorhabditis elegans: Evaluation of templates for homology-mediated repair and knock-ins by homology-independent DNA repair. G3: Genes, Genomes, Genetics 5, 1649-1656.

Kim, D.H., Grün, D., and Van Oudenaarden, A. (2013). Dampening of expression oscillations by synchronous regulation of a microRNA and its target. Nature Genetics 45, 1337-1345.

Koike, N., Yoo, S.H., Huang, H.C., Kumar, V., Lee, C., Kim, T.K., and Takahashi, J.S. (2012). Transcriptional architecture and chromatin landscape of the core circadian clock in mammals. Science 338, 349-354.

Krijthe, J.H. (2015). Rtsne: T-Distributed Stochastic Neighbor Embedding using a Barnes-Hut Implementation.

Lažetić, V., and Fay, D.S. (2017). Molting in C. elegans. Worm 6, e1330246.

McCarthy, D.J., Chen, Y., and Smyth, G.K. (2012). Differential expression analysis of multifactor RNA-Seq experiments with respect to biological variation. Nucleic Acids Research 40, 4288-4297.

Meeuse, M.W., Hauser, Y.P., Morales Moya, L.J., Hendriks, G., Eglinger, J., Bogaarts, G., Tsiairis, C., and Großhans, H. (2020). Developmental function and state transitions of a gene expression oscillator in Caenorhabditis elegans. Molecular Systems Biology 16.

Menet, J.S., Rodriguez, J., Abruzzi, K.C., and Rosbash, M. (2012). Nascent-Seq reveals novel features of mouse circadian transcriptional regulation. ELife 2012, 11.

Miki, T.S., Carl, S.H., and Großhans, H. (2017). Two distinct transcription termination modes dictated by promoters. Genes and Development 31, 1870-1879.

Minnich, M., Tagoh, H., Bönelt, P., Axelsson, E., Fischer, M., Cebolla, B., Tarakhovsky, A., Nutt, S.L., Jaritz, M., and Busslinger, M. (2016). Multifunctional role of the transcription factor Blimp-1 in coordinating plasma cell differentiation. Nature Immunology 17, 331-343.

Monsalve, G.C., and Frand, A.R. (2012). Toward a unified model of developmental timing. Worm 1, 221-230.

Moreno-Risueno, M.A., Norman, J.M. Van, Moreno, A., Zhang, J., Ahnert, S.E., and Benfey, P.N. (2010). Oscillating Gene Expression Arabidopsis Root Branching. Science 329, 1306-1312.

Moribe, H., Yochem, J., Yamada, H., Tabuse, Y., Fujimoto, T., and Mekada, E. (2004). Tetraspanin protein (TSP-15) is required for epidermal integrity in Caenorhabditis elegans. Journal of Cell Science 117, 5209-5220.

Oates, A.C., Morelli, L.G., and Ares, S. (2012). Patterning embryos with oscillations: Structure, function and dynamics of the vertebrate segmentation clock. Development 139, 625-639. analysis of larval developmental phenotypes in Caenorhabditis elegans. Genetics 201, 443-448. 
Panda, S., Hogenesch, J.B., and Kay, S.A. (2002). Circadian rhythms from flies to humans. Nature 417, 329-335.

Patke, A., Young, M.W., and Axelrod, S. (2020). Molecular mechanisms and physiological importance of circadian rhythms. Nature Reviews Molecular Cell Biology 21, 67-84.

Paus, R., and Foitzik, K. (2004). In search of the "hair cycle clock": A guided tour. Differentiation 72, 489-511.

Preußner, M., Goldammer, G., Neumann, A., Haltenhof, T., Rautenstrauch, P., Müller-McNicoll, M., and Heyd, F. (2017). Body Temperature Cycles Control Rhythmic Alternative Splicing in Mammals. Molecular Cell 67, 433-446.e4.

Robinson, M.D., McCarthy, D.J., and Smyth, G.K. (2009). edgeR: A Bioconductor package for differential expression analysis of digital gene expression data. Bioinformatics 26, 139-140.

Sandhu, A., Badal, D., Sheokand, R., Tyagi, S., and Singh, V. (2021). Specific collagens maintain the cuticle permeability barrier in Caenorhabditis elegans. Genetics 217.

Stec, N., Doerfel, K., Hills-Muckey, K., Ettorre, V.M., Ercan, S., Keil, W., and Hammell, C.M. (2021). An Epigenetic Priming Mechanism Mediated by Nutrient Sensing Regulates Transcriptional Output during C. elegans Development. Current Biology 31, 809-826.e6.

Stenn, K.S., and Paus, R. (2001). Controls of hair follicle cycling. Physiological Reviews 81, 449 494.

Takahashi, J.S. (2004). Introduction: Finding new clock components; past and future. 19, 339-347.

Telerman, S.B., Rognoni, E., Sequeira, I., Pisco, A.O., Lichtenberger, B.M., Culley, O.J., Viswanathan, P., Driskell, R.R., and Watt, F.M. (2017). Dermal Blimp1 Acts Downstream of Epidermal TGF $\beta$ and Wnt/ $\beta$-Catenin to Regulate Hair Follicle Formation and Growth. Journal of Investigative Dermatology 137, 2270-2281.

Tsiairis, C., and Großhans, H. (2020). Gene expression oscillations in C. elegans underlie a new developmental clock. (Academic Press), p.

Valentine, J.W., and Collins, A.G. (2000). The significance of moulting in Ecdysozoan evolution. Evolution and Development 2, 152-156.

Zhang, L., Ward, J.D., Cheng, Z., and Dernburg, A.F. (2015). The auxin-inducible degradation (AID) system enables versatile conditional protein depletion in C. elegans. Development 142, 4374-4384.

Zhang, Y., Liu, T., Meyer, C.A., Eeckhoute, J., Johnson, D.S., Bernstein, B.E., Nussbaum, C., Myers, R.M., Brown, M., Li, W., et al. (2008). Model-based analysis of ChIP-Seq (MACS). Genome Biology 9, R137.

Acknowledgements: We thank Gert-Jan Hendriks for advice and discussion during the conception of this project, Stephane Thiry, Kirsten Jacobeit and the FMI Functional

Genomics Facility for RNA sequencing, Iskra Katic for help in generating transgenic strains, Kathrin Braun for help with the Western Blots, Sarah H. Carl for help with RNA Pol II ChIPseq analysis, Lukas Burger and Michael Stadler for input on ChIP-seq analysis, Laurent 
Gelman for pivotal help with imaging, and Charisios Tsiairis and Benjamin Towbin for a critical reading of the manuscript. Funding: The FMI is core-funded by the Novartis Research Foundation. This work has received funding from the European Research Council (ERC) under the European Union's Horizon 2020 research and innovation programme (grant agreement No [741269], to H.G.). M.W.M.M. is a recipient of a Boehringer Ingelheim Fonds PhD fellowship. Author contributions: Milou Meeuse performed the RNAPII ChIP sequencing time course. Yannick Hauser performed all transcriptional reporter RT-qPCR time courses. Milou Meeuse and Yannick Hauser performed luciferase assays with blmp-1 RNAi and mutant animals respectively. Milou Meeuse performed the auxin inducible degron luciferase assays. Yannick Hauser performed and analyzed the blmp-1(tm548) mutant time course and acquired and analyzed single worm imaging data. Helge Großhans, Milou Meeuse and Yannick Hauser conceived the project and Yannick Hauser and Helge Großhans wrote the manuscript. Competing interests: The authors declare no competing interests. Data and materials availability: All sequencing data generated for this study have been deposited in NCBI's Gene Expression Omnibus (Edgar, 2002) and are accessible through GEO Series accession number GSE169642 (PolII ChIP-sequencing and RNA-sequencing), GSE141514 (blmp-1(tm548) RNA-sequencing), GSE163461 (N2 and blmp-1(tm548) RNA-sequencing, $2^{\text {nd }}$ replicate). GSE147388 (N2 RNA-sequencing) provides a wild-type time course collected and sequenced in parallel to GSE141514 that was published previously (Meeuse et al., 2020).

20 Published research reagents from the FMI are shared with the academic community under a Material Transfer Agreement (MTA) having terms and conditions corresponding to those of the UBMTA (Uniform Biological Material Transfer Agreement). 
A

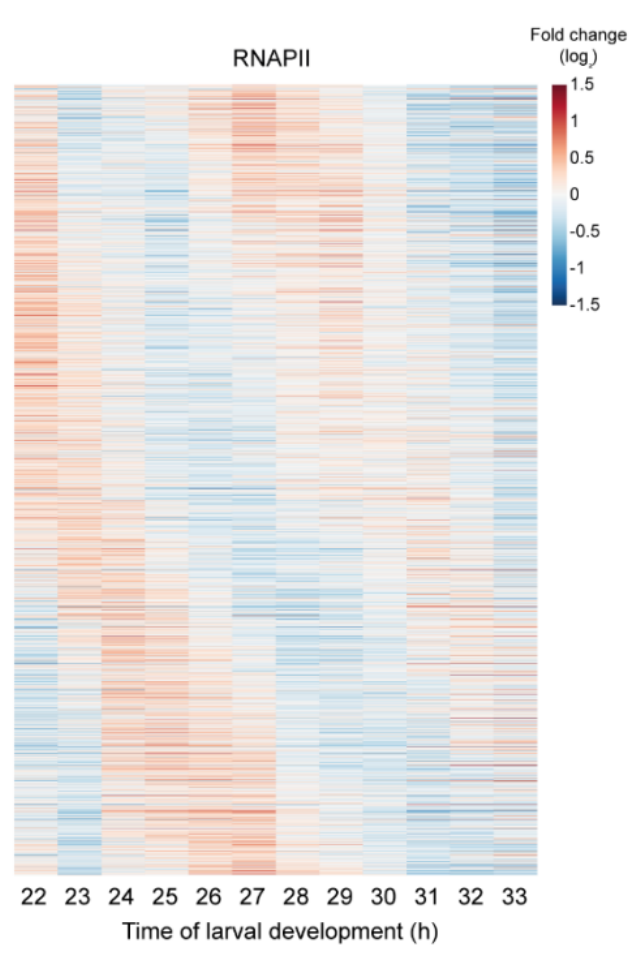

C
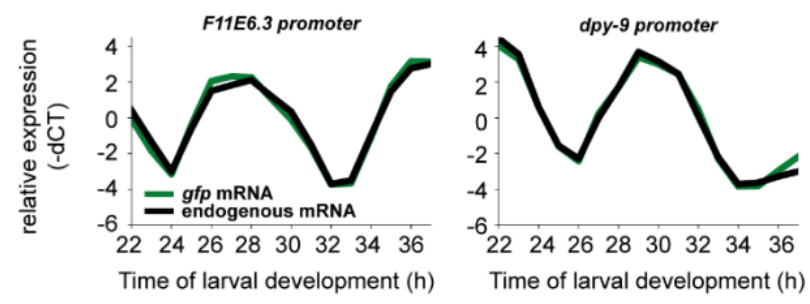

B
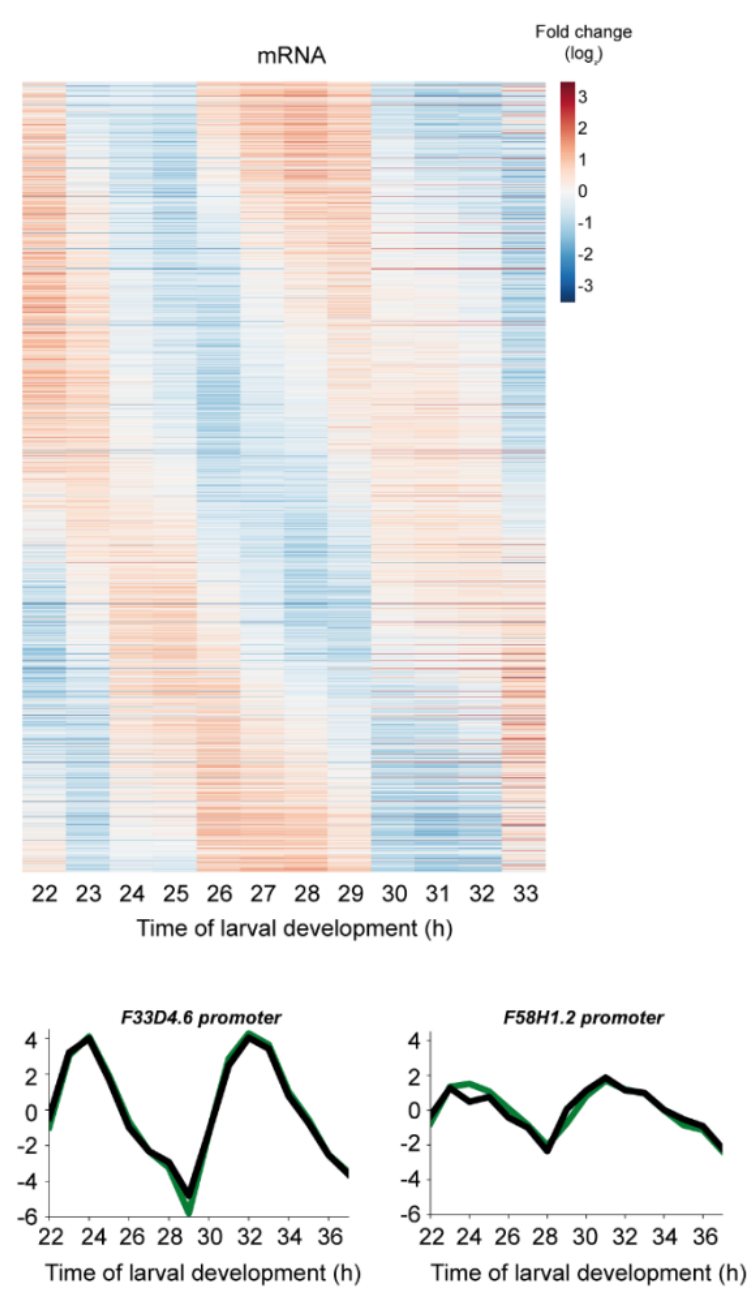

Fig 1: Oscillatory gene expression arises from promoter-driven rhythmic transcription

A, B, Log2-transformed, mean-normalized RNA polymerase II ChIP (A) and RNA (B) sequencing reads of detectably expressed oscillating genes $(n=2,106)$. Reads are ordered according to peak phase obtained from Meeuse et al., 2020.

C, RT-qPCR time courses. Promoters of the indicated oscillating genes were used to drive expression of a destabilized, nuclear GFP from a single copy integrated transgene; $g f p$ and the endogenous transcript driven by the same promoter were quantified from the same RNA samples. Relative expression was plotted as (target $\mathrm{Ct}$ values - actin $\mathrm{Ct}$ values) * (-1) and then mean normalized for each trace individually $(\mathrm{C})$.

See also Figure $\mathrm{S} 1 \&$ S2. 


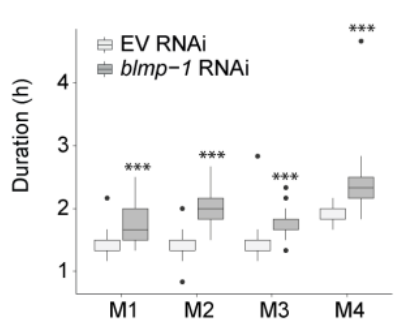

D

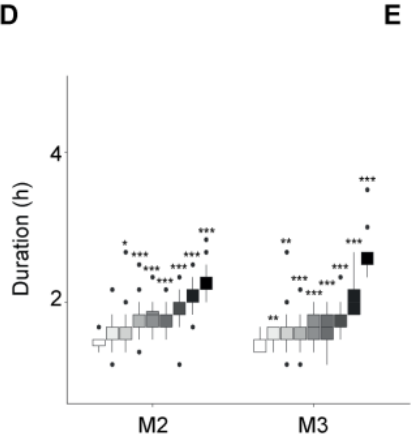

E

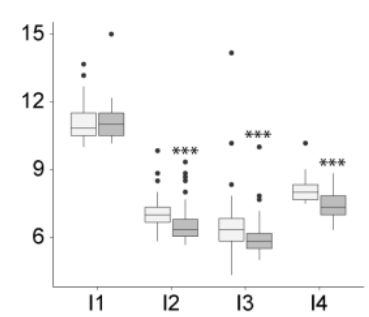

C

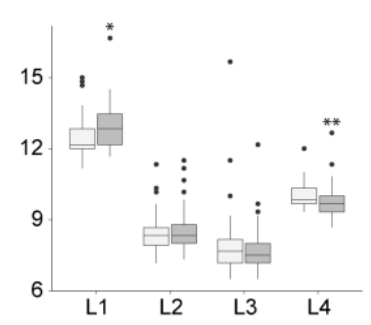

$\mathbf{F}$

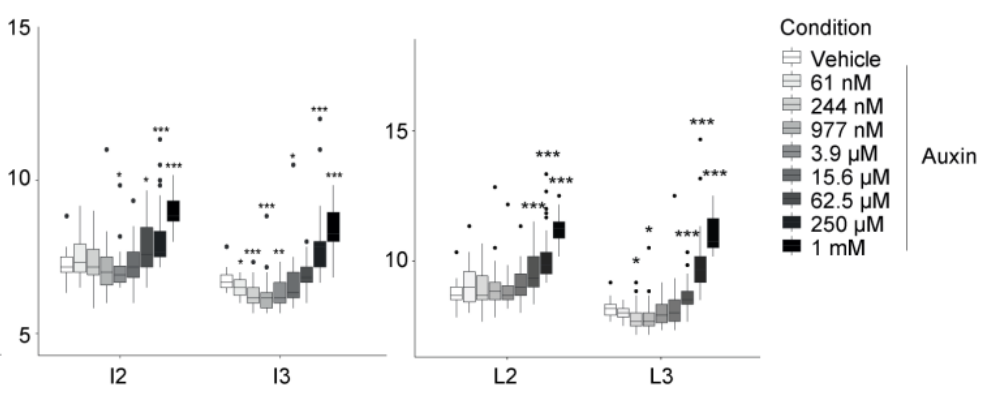

G

H

I
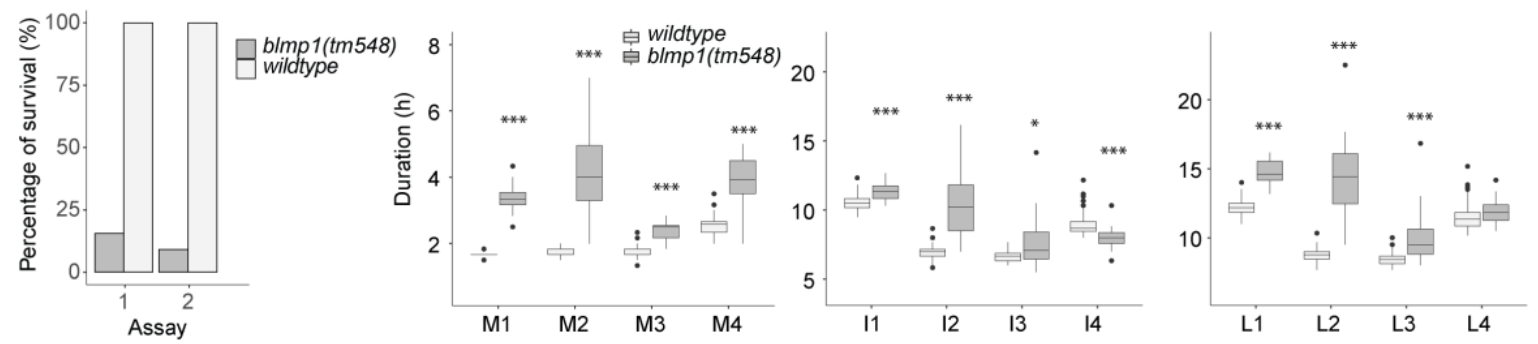

Fig 2: BLMP-1 loss of function leads to developmental defects

A - C, Boxplots of molt (A), intermolt (B) and larval stage (C) durations in mock $(n=59)$ and blmp-1 RNAi-treated animals $(\mathrm{n}=50)$ as determined by a single-animal luciferase assay. Significantly different durations are indicated $(* \mathrm{P}<0.05$, ** $\mathrm{P}<0.01$, *** $\mathrm{P}<0.001$, Welch two-sample and two-sided t-test)

D - F, Boxplots of molt (A), intermolt (B) and larval stage (C) durations of aid::blmp-1 animals exposed to vehicle or auxin at the indicated concentration from egg stage to adulthood.

G, Bar plot of manual quantification of lethality or arrest detected in two replicate luciferase assays for wild-type (replicate $1: \mathrm{n}=76$, replicate $2: \mathrm{n}=80$ ) and $t m 548(\operatorname{tm} 548$ ) worms (replicate $1: n=129$, replicate $2: n=77$ ), respectively. 
$\mathbf{H}$ - J Boxplots of single animal molt (H), intermolt (I) and larval stage (J) durations of wildtype $(\mathrm{n}=76)$ and $\operatorname{blmp}$-1(tm548) $(\mathrm{n}=20)$ mutant animals. blmp-1(tm548) animals are the surviving individuals from assay $\# 1$ in $(G)(n=20)$.

Significantly different durations are indicated in $(\mathrm{A})-(\mathrm{F})$ and $(\mathrm{H})-(\mathrm{J}),{ }^{*} \mathrm{P}<0.05,{ }^{*} * \mathrm{P}<0.01$, $* * * \mathrm{P}<0.001$, Wilcoxon unpaired, two-sample test. In the boxplots, the horizontal line represents the median, hinges extend to first and third quartiles and the whiskers extend up to $1.5^{*} \mathrm{IQR}$ (interquartile range). Data beyond that limit is plotted as dots.

See also Figure S3. 
A

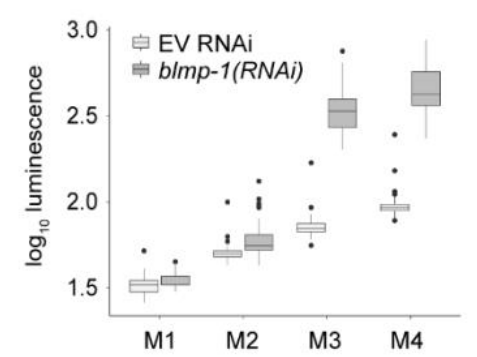

D

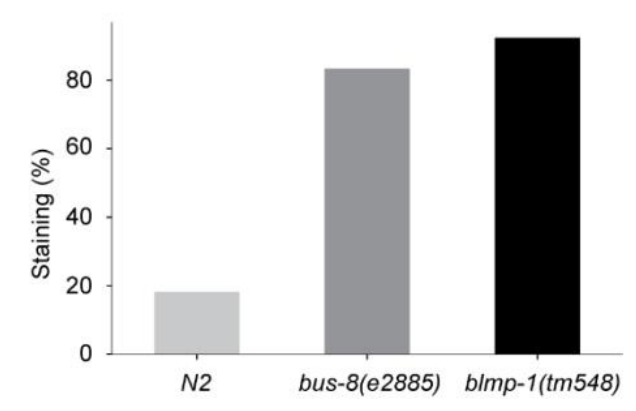

C
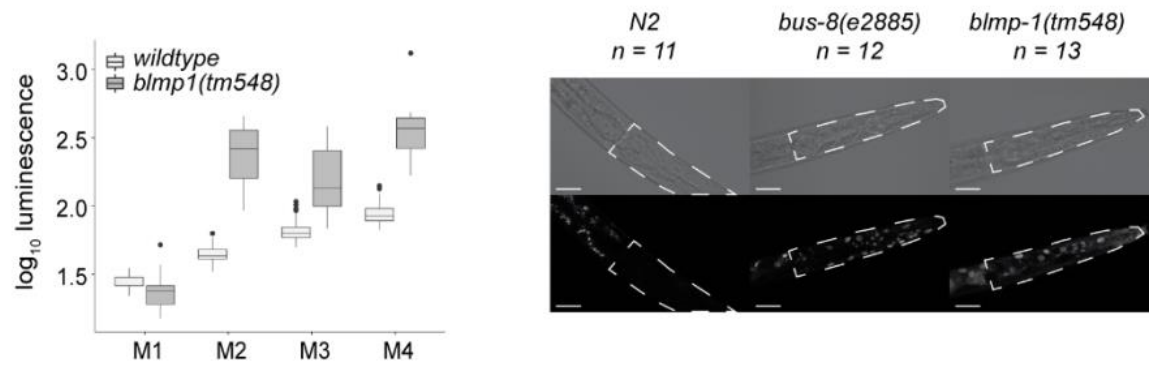

Fig 3: BLMP-1 is required for cuticle integrity

A, B, Boxplots showing $\log _{10}$-transformed luminescence intensities during the indicate molts in mock (EV) and blmp-1 RNAi (A) and in wild-type and blmp-1(tm548) mutants (B).

C, D, Animals of the indicated genotypes were incubated with Hoechst 33258 to test for cuticle permeability Representative images of (C) and quantification of the percentage of stained worms (D) are shown. A dashed white line in (C) outlines the head region used for scoring; note that signal outside this area in N2 corresponds to autofluorescence. Scale bars in (C) represent $20 \mu \mathrm{m}, \mathrm{n} \geq 11$ in (D)

Boxplots in (A), (B) represent the median as horizontal line and hinges extend to first and third quartiles, the whiskers extend up to $1.5 * \mathrm{IQR}$ (interquartile range). Data beyond that limit is plotted as dots. 
A

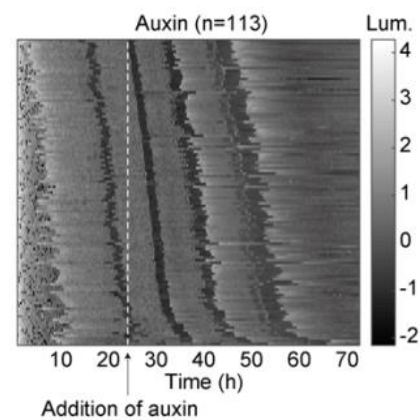

B

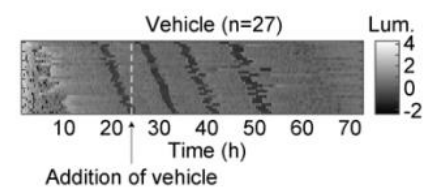

Addition of auxin
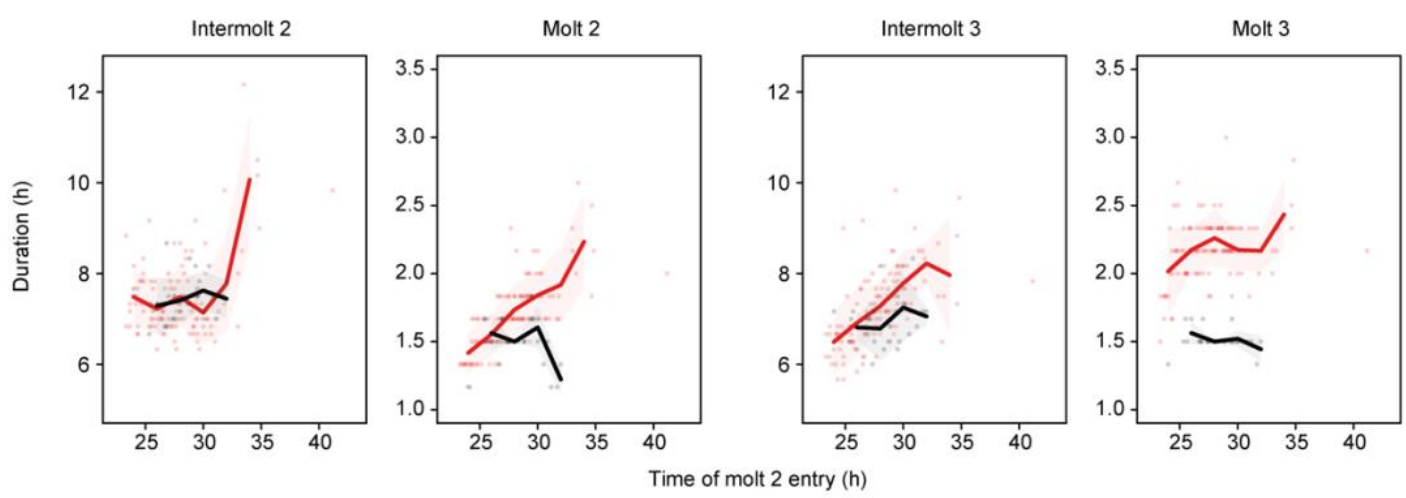

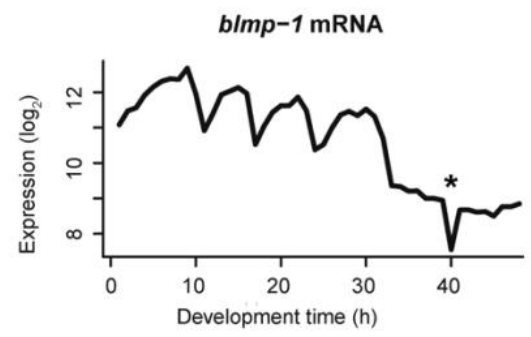

D

Developmental Time (h)

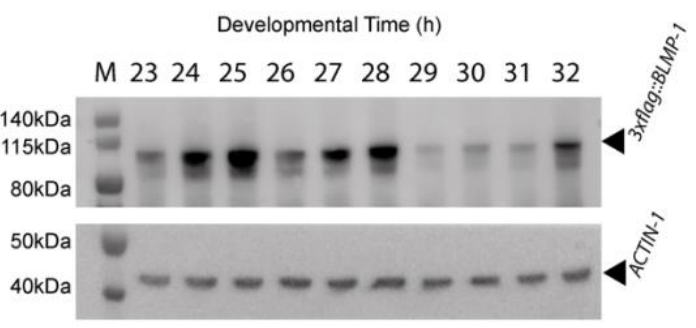

E Developmental Time (h)

IM2 $\mathrm{M} 2$

M $17 \quad 181920212223242526 \quad 27$

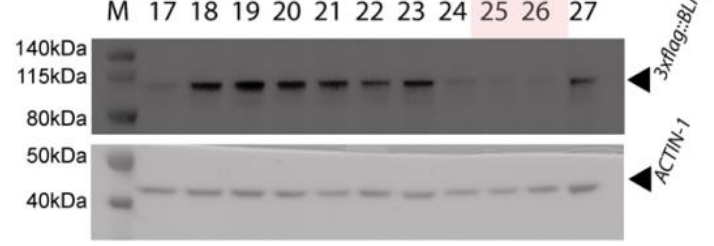

Fig 4: Rhythmic activity and accumulation of BLMP-1

A, Heatmaps showing trend-corrected luminescence (Lum.) traces; one animal per horizontal line. Vehicle ( $0.25 \%$ ethanol; left) or $250 \mu \mathrm{M}$ auxin (right) were added at $\mathrm{t}=24 \mathrm{~h}$ after placing single embryos into wells. $\mathrm{t}=0 \mathrm{~h}$ corresponds to the start of the luminescence assay. Embryos hatch at different time points and traces are sorted by entry into second molt. Dark gray indicates low luminescence and corresponds to the molts. 
B, Duration of intermolt 2, molt 2, intermolt 3 and molt 3 plotted over the time of molt entry 2 relative to auxin treatment in control and auxin treated animals shown in A. The line represents the mean and shading indicates the standard deviation.

C, blmp-1 mRNA levels oscillate during larval development. Data from (Meeuse et al., 2020), with populations of synchronized animals grown at $25^{\circ} \mathrm{C}$ sampled hourly. An asterisk marks an outlier timepoint.

D, E, 3xFLAG::BLMP-1 protein detected by Western Blot. Protein abundance covering roughly L3 to early $\mathrm{L} 4\left(25^{\circ} \mathrm{C}\right)$ using HW2639 (D) and from early L2 to early L3 stage $\left(20^{\circ} \mathrm{C}\right)$ using HW2802 (E). The molt in E is determined by luciferase assays that were performed in parallel (Fig S9) and indicated in salmon.

See also Figure S4, S9 
bioRxiv preprint doi: https://doi.org/10.1101/2021.07.05.450828; this version posted July 5, 2021. The copyright holder for this preprint (which was not certified by peer review) is the author/funder, who has granted bioRxiv a license to display the preprint in perpetuity. It is made available under aCC-BY-NC 4.0 International license.

A

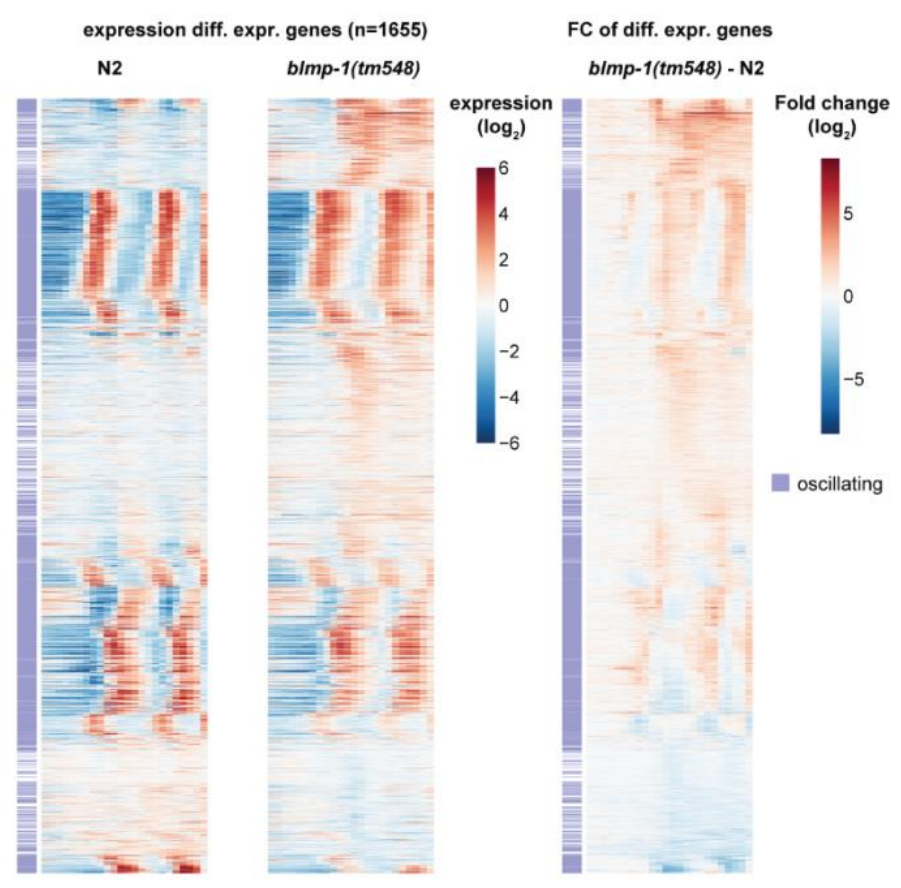

C
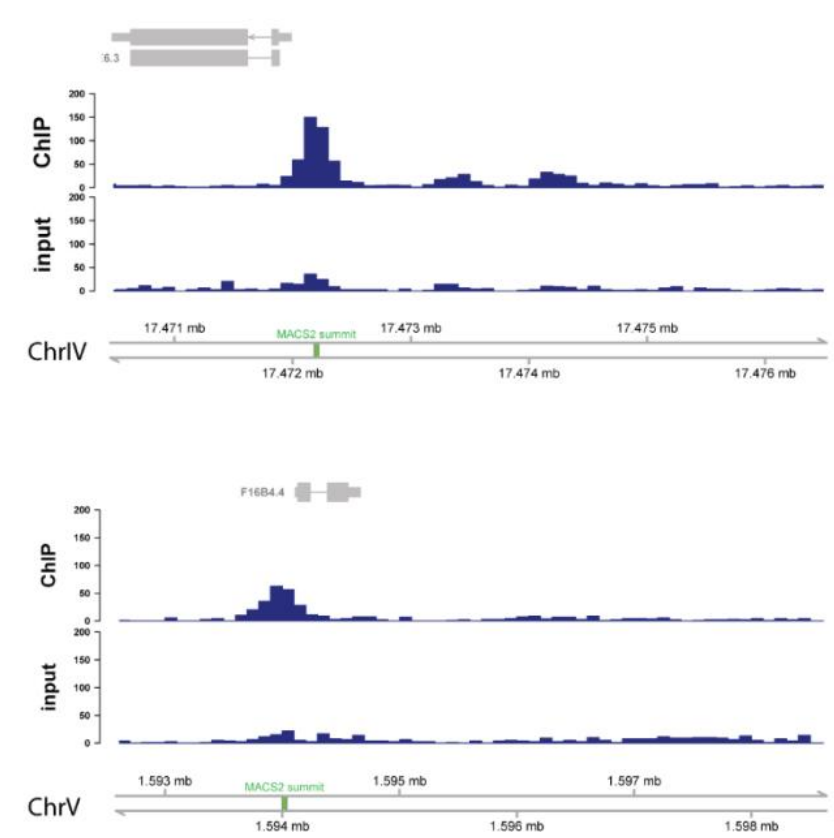

B

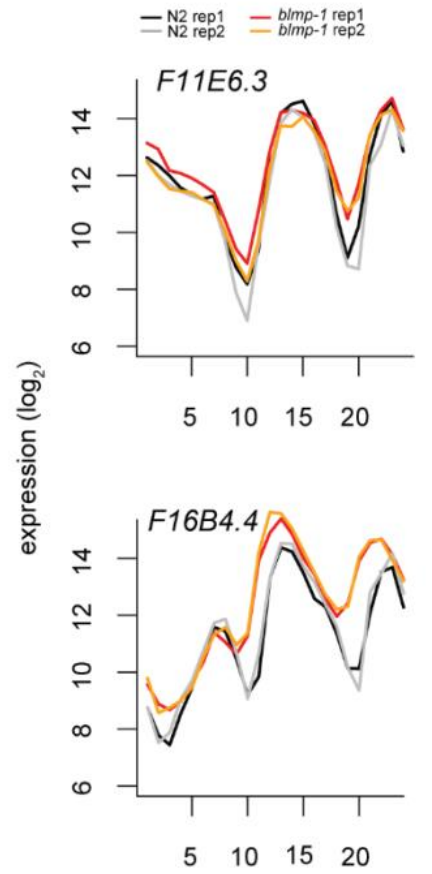

D

Realigned time after plating
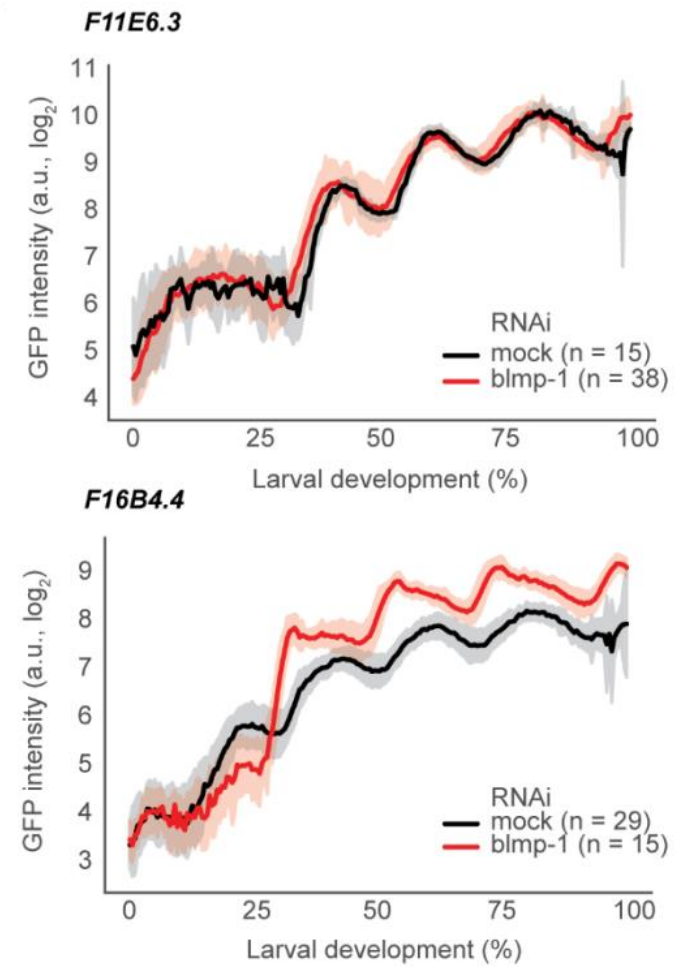

Fig 5: Time-course analysis reveals genes with dysregulated expression in blmp-1(tm548) animals 
A, Heatmap of $\log _{2}$-transformed gene expression and fold changes of differentially expressed genes in realigned blmp-1 mutant RNA sequencing time course. The heatmap was ordered according to a 1-dimensional tSNE (see Methods).

B, $\log _{2}$-transformed expression levels over realigned time for F11E6.3 and F16B4.4 transcripts for the indicated strains and replicates from RNA-sequencing time courses.

C, BLMP-1 ChIP-seq binding profiles for the promoters of the genes shown in (B). The binding peak identified by MACS2 analysis is indicated in green.

D, Single worm GFP intensities of transcriptional reporters based on the promoters of the genes in (B) were scaled to larval development (hatch until molt 4 exit, see methods) and $\log _{2}$-transformed for mock (black) and blmp-1 (red) RNAi-treated animals. The indicated number of single animals were followed over time; GFP intensities were averaged and plotted with the respective standard deviations in shading.

See also Figures S6, S7 
A

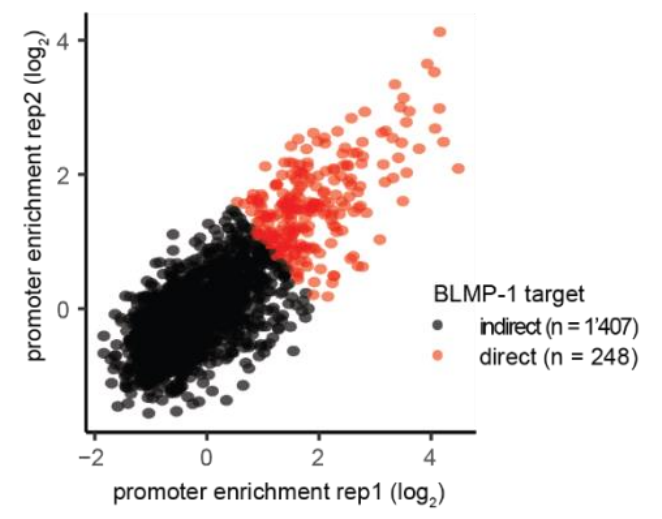

D
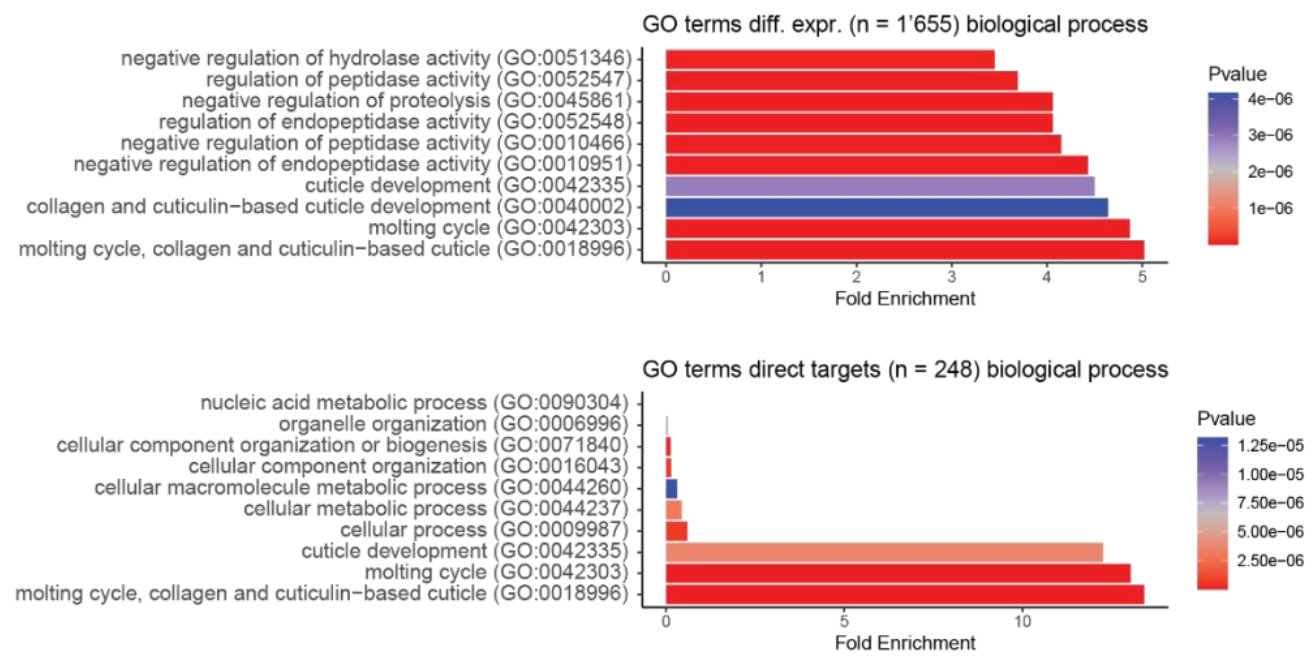

Fig 6: Direct BLMP-1 targets function in molting

A, Scatter plot showing promoter-binding of BLMP-1 as $\log _{2}$-fold enrichment over input in two replicate ChIP-Seq experiments for all genes that significantly change between blmp$1($ tm548) and wild-type animals, shown in Fig 5A. Every dot is a gene, red indicates binding enrichment across replicates at a $\log _{2}$ average of $>1$.

B - C, Fraction of direct target genes (B) and 400 non-significantly changed genes (selected by highest p-values) in the RNA sequencing time course $(\mathrm{C})$ that are expressed at sufficiently 
correlation (r) of blmp- 1 expression with the fraction of genes significantly expressed in cells or tissues is indicated above the plot.

D, Bar plots showing enrichment of gene ontology (GO) terms related to biological processes for genes differentially expressed in blmp-1(tm548) mutant animals (top, as in Fig. 5A) and

5 direct BLMP-1 targets (bottom; as in panel (A)). The top 20 most highly enriched terms were sorted according to P-value followed by plotting the most 10 significant terms.

See also Figure S8 
bioRxiv preprint doi: https://doi.org/10.1101/2021.07.05.450828; this version posted July 5, 2021. The copyright holder for this preprint (which was not certified by peer review) is the author/funder, who has granted bioRxiv a license to display the preprint in perpetuity. It is made available under aCC-BY-NC 4.0 International license.

A

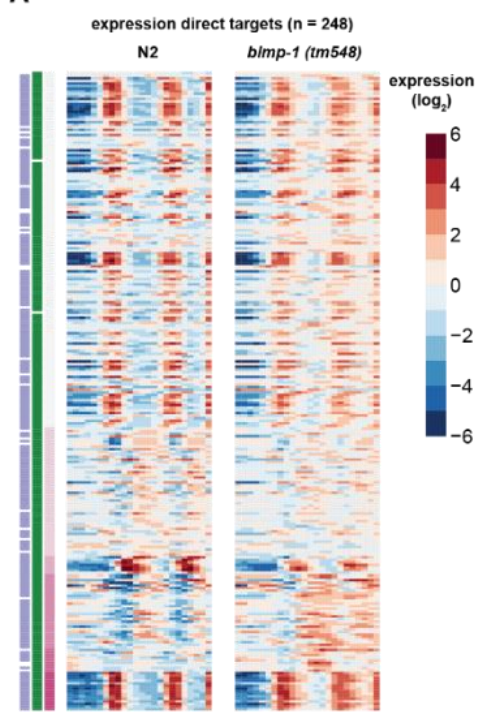

FC direct targets

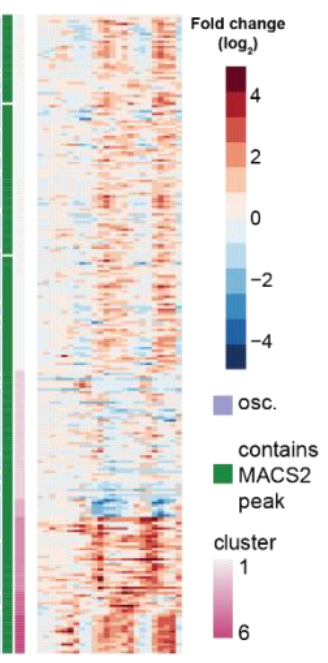

B
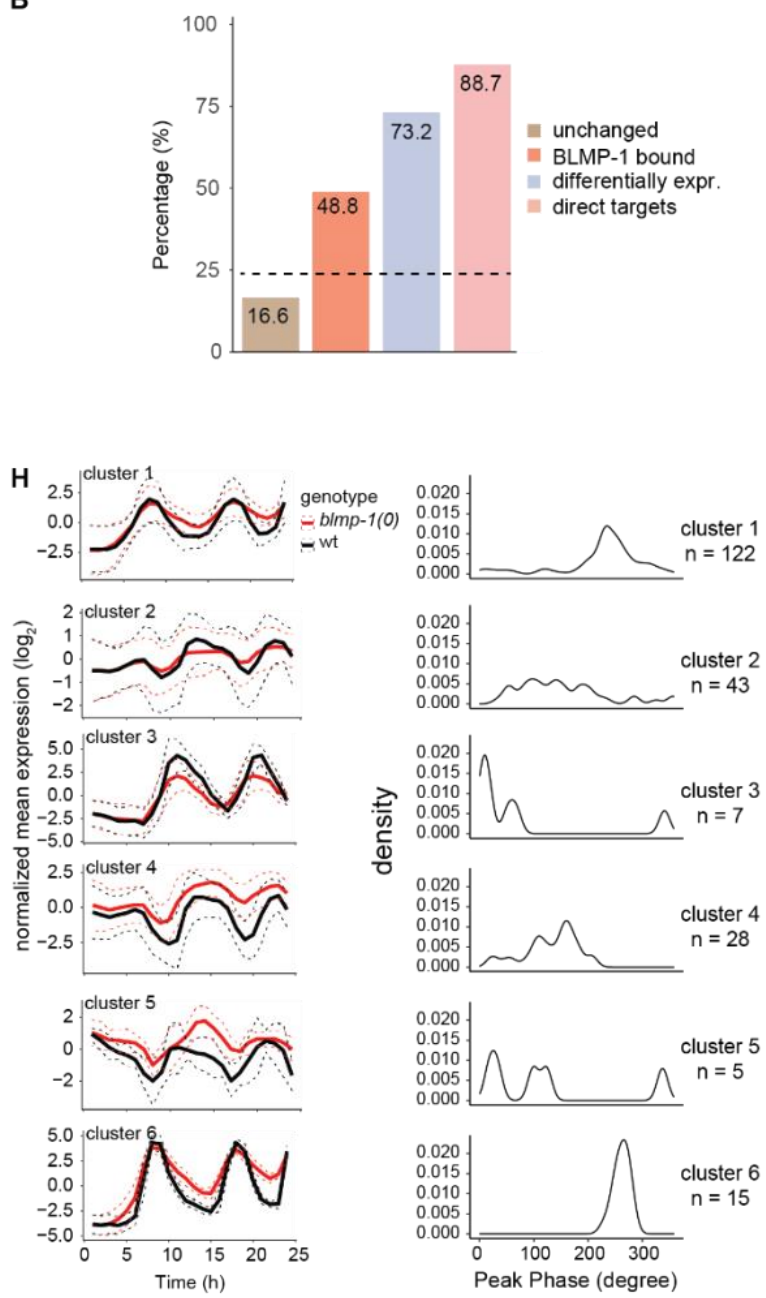

C
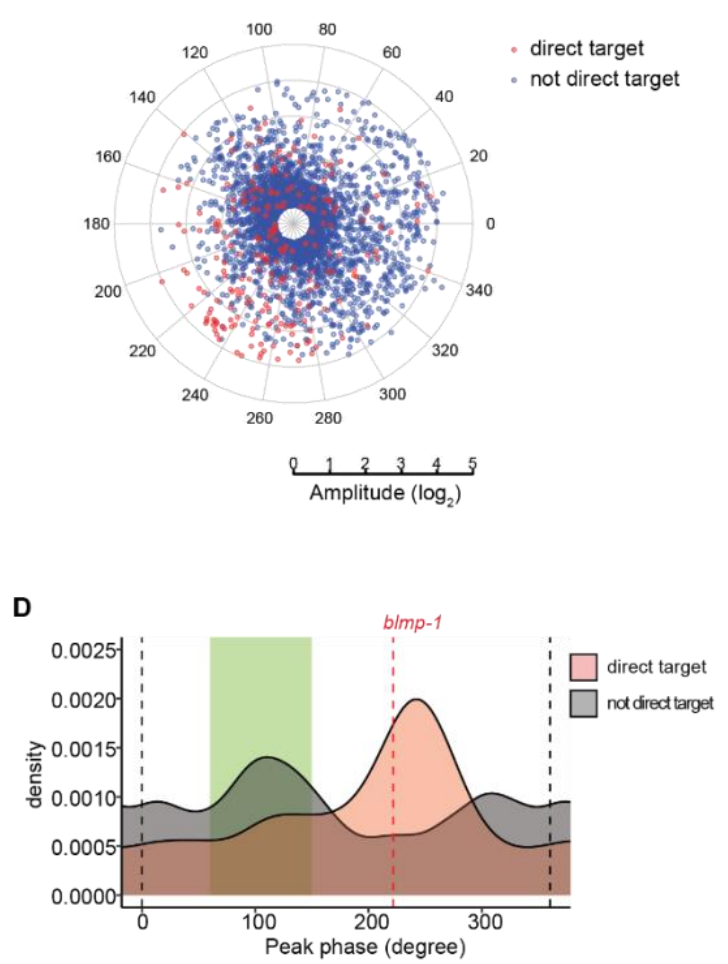

E

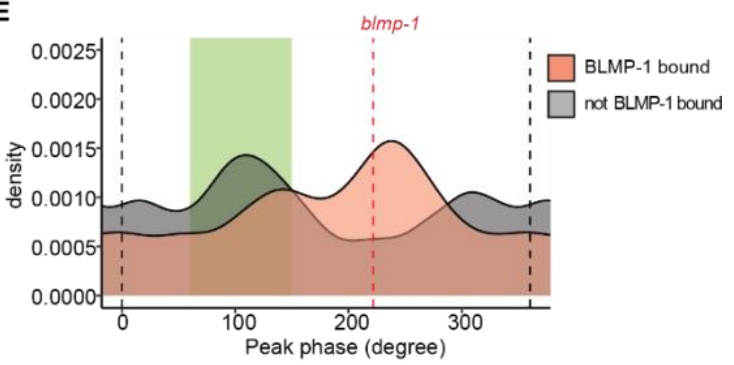

$\mathbf{F}$
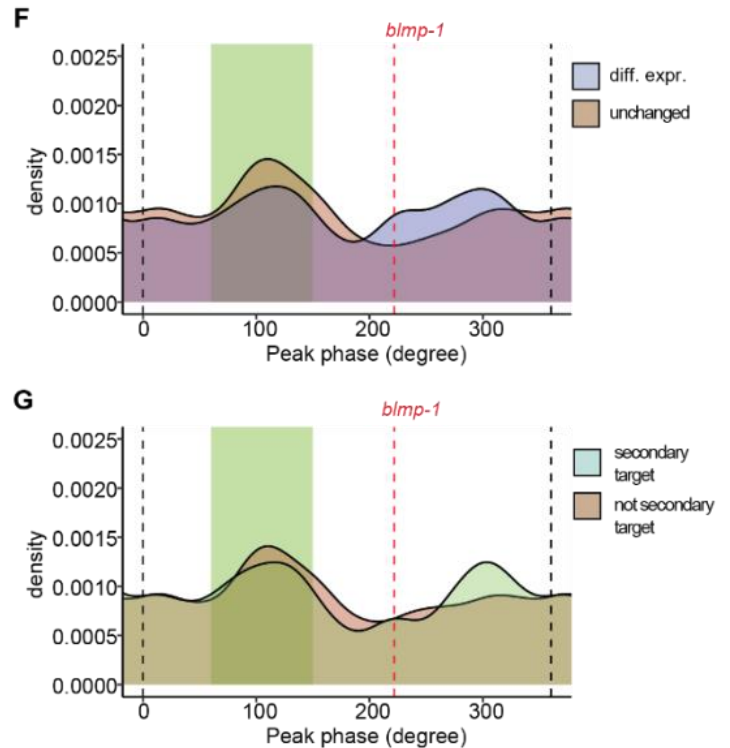

Fig 7: BLMP-1 targets shapes oscillating gene expression of direct targets 
A, Heatmap of $\log _{2}$-transformed gene expression and fold changes of direct BLMP-1 targets as identified in Fig 6A. The heatmaps were all ordered according to hierarchical clustering based on the fold changes and cut into 6 different clusters, indicated in shades of purple.

B, Percentage of oscillating genes among the indicated categories of genes. Categories are (right to left) "Direct targets" according to Fig 6A, "diff. expr." (differentially expressed) according to Fig 5A, "BLMP-1-bound" according to Fig S5, and "unchanged", which are all expressed genes used as input for differential expression analysis (see Methods) except those that are differentially expressed according to Fig 5A. Dashed line indicates the expected fraction of oscillating genes according to (Meeuse et al., 2020)

C, Polar plot displaying the peak phase (circular axis, degrees) and the amplitude (radial axis, $\log _{2}$ ) of oscillating genes. The direct targets according to Fig 6A are marked in red.

D-G, Peak phase distribution of oscillating genes among the indicated categories (as detailed in B). The phase of the molt is indicated in green. The dotted line at $0^{\circ}$ and $360^{\circ}$ indicate the boundaries of the circular phase information $\left(360^{\circ}=0^{\circ}\right)$. The peak phase of blmp-1 mRNA is represented with the red dotted line.

H, Mean-normalized mean expression and peak phase distributions of oscillating genes identified in the 6 clusters in A. The standard deviation is shown in dashed lines.

See also Fig. S9 


\section{Methods}

\section{RNA polymerase II ChIP-sequencing}

For RNA polymerase II ChIP-sequencing, synchronized L1 wild-type worms were grown at $25^{\circ} \mathrm{C}$. Worms were collected hourly from 22 hours (90.000 worms) until 33 hours (46.000 worms) developmental time. RNA polymerase II ChIP was performed as previously described (Miki et al., 2017). In short, worms were incubated in M9 with 2\% formaldehyde for 30 minutes at room temperature with gentle agitation to allow protein-DNA crosslinking. Worms were lysed with beads using the FastPrep-24 5G machine (MP Biomedicals, settings: $8 \mathrm{~m} / \mathrm{sec}, 30 \mathrm{sec}$ on, $90 \mathrm{sec}$ off, 5 cycles). Lysates were sonicated using the Bioruptor Plus Sonication system (Diagenode, settings: $30 \mathrm{sec}$ on, $30 \mathrm{sec}$ off, 20 cycles). $250 \mu \mathrm{g}$ sonicated chromatin was incubated with $10 \mu \mathrm{g}$ mouse anti-RNA polymerase II CTD antibody (8WG16, Abcam) at $4{ }^{\circ} \mathrm{C}$ for 2 hours with gentle agitation and subsequently with $45 \mu \mathrm{L}$ Dynabeads Protein $\mathrm{G}$ (Thermo Fisher Scientific) at $4{ }^{\circ} \mathrm{C}$ overnight with gentle agitation. Eluate was treated with $0.13 \mu \mathrm{g} / \mu \mathrm{l}$ RNase and $1 \mu \mathrm{g} / \mu \mathrm{l}$ Proteinase K. ChIP-seq libraries were prepared using NEBNext Ultra DNA Library Prep Kit for Illumina (New England Biolabs) and sequenced using the HiSeq 50 cycle single-end reads protocol on the HiSeq 2500 system.

Sequencing reads were aligned to the ce10 $C$. elegans genome using the qAlign function (default parameters) from the QuasR package (Gaidatzis et al., 2015) in R. ChIP-seq counts within $1-\mathrm{kb}$ windows, i.e. $-500 \mathrm{bp}$ to $+500 \mathrm{bp}$ around the annotated TSS (using WS220/ce10 annotations), were scaled by total mapped library size per sample and $\log _{2}$-transformed after adding a pseudocount of 8 . Genes with a mean scaled TSS window count of less than 8 across all samples were excluded. $\log _{2}$-transformed counts were then quantile-normalized using the normalize.quantiles function from the preprocessCore library (Bolstad, 2021; Bolstad et al., 2003) in R. Finally, quantile-normalized values were row-centered. 


\section{RNA-sequencing for PolII ChIP-seq}

For total RNA sequencing, worms were collected hourly from 22 hrs (10.000 worms) until 33 hrs (5.000 worms per time point) after plating from the same plates as for ChIP sequencing. RNA was extracted in Tri Reagent and DNase treated as described previously (Hendriks et al., 2014). Total RNA-seq libraries were prepared using Total RNA-seq ScriptSeq Library Prep Kit for Illumina (New England Biolabs) and sequenced using the HiSeq 50 cycle singleend reads protocol on the HiSeq 2500 system. RNA-seq data were mapped to the $C$. elegans genome (ce10) using the qAlign function (splicedAlignment=TRUE, Rbowtie aligner version 1.16.0) from the QuasR package in R (Au et al., 2010; Gaidatzis et al., 2015; Kim et al., 2015; Langmead et al., 2009) (version 1.16.0). Exonic expression was quantified using qCount function from the QuasR package in $\mathrm{R}$ and the exon annotation of the ce10 assembly (version WS220). Counts were scaled by total mapped library size for each sample. A pseudocount of 8 was added and counts were log2-transformed. Lowly expressed genes were excluded (maximum $\log 2$-transformed exonic expression - (log2(gene width) mean $(\log 2($ gene width $))) \leq 6)$. Of the previously annotated 'high-confidence-oscillating' genes ( $n=3,739)$ (Meeuse et al., 2019), 2,106 genes were sufficiently expressed on the exonic level at the chosen sequencing depth. Oscillating genes were sorted by phase and meannormalized expression was plotted in heatmaps.

\section{RT-qPCR reporters}

Gravid adult worms were bleached to specifically obtain eggs which were incubated in M9 buffer overnight (12 to 16 hours) on a rotating wheel. After incubation, hatched worms will be synchronized in L1 arrest due to starvation. The synchronized L1 population was plated onto agar plates with food (E. coli, OP50) to initiate synchronous larval development. The concentration of worms per plate can vary between 1'000 and 4'000 worms per plate. In 
total, 2’000 - 8'000 worms were sampled each time point with fewer worms for the last time points.

Worms were collected hourly between 22 and 37 hours at $25^{\circ} \mathrm{C}$ (for $g f p$ reporter data) after plating synchronized L1. Worms were washed off the plate(s) and washed 3 times in M9 buffer. After washing, $1 \mathrm{ml}$ Tri Reagent (MRC) was added. Samples were frozen in liquid nitrogen and stored overnight at $-80^{\circ} \mathrm{C}$. Conventional RNA isolation using phenol chloroform extraction (adapted from (Bethke et al., 2009) was used to extract RNA which was then diluted to the same concentration for each sample and used as input for the Promega Protocol: "ImProm-IITM Reverse Transcription System" to convert RNA to cDNA. The resulting cDNA was diluted 1:1000 to quantify actin transcript levels and 1:20 for endogenous transcripts. qPCR was then performed on a Step one Realtime PCR machine using primer pairs of which one was exon-exon spanning to detect mature mRNA levels of endogenous genes corresponding to the reporters and the $g f p$ transcript.

\section{RT-qPCR primers:}

\begin{tabular}{|c|c|c|c|}
\hline Transcript & $\begin{array}{c}\text { Primer } \\
\text { name }\end{array}$ & Description & Sequence \\
\hline \multirow{2}{*}{$g f p$} & YPH120 & $\begin{array}{l}\text { RV exon-exon spanning } \\
\text { primer }\end{array}$ & ACAAGTGTTGGCCATGGA \\
\hline & YPH121 & FW primer & CTTGTTGAATTAGATGGTGATGTT \\
\hline \multirow[b]{2}{*}{ F58H1.2 } & YPH126 & FW primer & TGATGTCGTCCATGGGAT \\
\hline & YPH127 & $\begin{array}{l}\text { RV exon-exon spanning } \\
\text { primer }\end{array}$ & CCATACGTATCCATTCCCA \\
\hline \multirow[b]{2}{*}{$R 12 E 2.7$} & YPH128 & FW primer & TCTTCTCTGCTTCTGCTT \\
\hline & YPH129 & $\begin{array}{l}\text { RV exon-exon spanning } \\
\text { primer }\end{array}$ & CTCCTCCGCATGGGT \\
\hline \multirow[t]{2}{*}{ F11E6.3 } & YPH164 & $\begin{array}{l}\text { FW exon-exon spanning } \\
\text { primer }\end{array}$ & CCCATCCGATGAAACGTCA \\
\hline & YPH165 & RV primer & TGGGGCGGTTTCTTCTTGA \\
\hline \multirow{2}{*}{ F33D4.6 } & YPH166 & $\begin{array}{l}\text { FW exon-exon spanning } \\
\text { primer }\end{array}$ & CCСТCCAATGATCAACTTG \\
\hline & YPH167 & $\mathrm{RV}$ primer & ATGAATCTTTCGTCTTGGAAGG \\
\hline \multirow{2}{*}{ C05C10.3 } & YPH168 & $\begin{array}{l}\text { FW exon-exon spanning } \\
\text { primer }\end{array}$ & TTAGTTGGCGGCTTCGGA \\
\hline & YPH169 & $\mathrm{RV}$ primer & GTCGAGTTTGAAGGAGCAAG \\
\hline$d a f-6$ & YPH170 & $\begin{array}{l}\text { FW exon-exon spanning } \\
\text { primer }\end{array}$ & CTATCACGAGGCCTTTCCA \\
\hline
\end{tabular}




\begin{tabular}{|c|c|l|l|}
\hline & YPH171 & RV primer & CCCCACAACGTCATATAACCAAA \\
\hline \multirow{3}{*}{ col-10 } & YPH430 & $\begin{array}{l}\text { FW exon-exon spanning } \\
\text { primer }\end{array}$ & GGTTCACGATGAGGTTCT \\
\cline { 2 - 4 } & YPH431 & RV primer & GTTGAATGGGTTGACACG \\
\hline \multirow{3}{*}{$d p y-9$} & YPH544 & $\begin{array}{l}\text { FW exon-exon spanning } \\
\text { primer }\end{array}$ & GTAGAGTTGTGTAAGACCGAG \\
\cline { 2 - 4 } & YPH545 & RV primer & GAGTACAAGCACAGCAGG \\
\hline \multirow{3}{*}{ act-1 } & $\begin{array}{c}a c t-1 \mathrm{FW} \\
\text { qPCR }\end{array}$ & FW primer & GTTGCCCAGAGGCTATGTTC \\
\cline { 2 - 4 } & $\begin{array}{c}a c t-1 \text { RV } \\
\text { qPCR }\end{array}$ & RV primer & CAAGAGCGGTGATTTCCTTC \\
\hline
\end{tabular}

\section{RT-qPCR analysis}

From two technical replicates, the mean actin $\mathrm{Ct}$ values were subtracted from the mean target $\mathrm{Ct}$ values, to obtain a relative quantification, represented by delta $\mathrm{Ct}(\mathrm{dCt})$. To obtain the mean normalized mRNA levels, the $\mathrm{dCt}$ mean of the time series was subtracted from from each time point value first and then multiplied by -1 . These values were then plotted to compare endogenous versus gfp mRNA levels.

\section{Luciferase assays}

Embryos were obtained by bleaching gravid adults that express the xeSi296 transgene [eft3p::luc::gfp::unc-54 3'UTR, unc-119(+)] II obtained by single-copy integration into the oxTi185 locus on chromosome II. Single embryos were transferred by pipetting into a well of a white, flat-bottom, 384-well plate (Berthold Technologies, 32505) and hatched in $90 \mu 1$ liquid culture. For RNAi experiments, the feeding method was used. E. coli HT115 bacteria carrying empty plasmids (L4440, mock RNAi) or an RNAi plasmid with an insert targeting blmp-1 (Fraser et al., 2000; Kamath and Ahringer, 2003) were induced with 1 mM IPTG for 1 hour at $37^{\circ} \mathrm{C}$. For blmp-1 mutant luciferase assays, OP50 was used instead of HT115 bacteria. Bacteria were diluted in S-Basal medium (OD $600=0.9)$, with $100 \mu \mathrm{M}$ firefly Dluciferin, $100 \mu \mathrm{g} / \mathrm{mL}$ ampicillin and $1 \mathrm{mM}$ IPTG in the case of RNAi. For Auxin Inducible Degradation (AID) experiments, $E$. coli OP50 were diluted in S-Basal medium $\left(\mathrm{OD}_{600}=0.9\right)$ 
and $100 \mu \mathrm{M}$ Firefly D-Luciferin (p.j.k., 102111). 3-Indoleacetic acid (Auxin, Sigma-Aldrich, I2886) was dissolved in $100 \%$ ethanol and diluted 400 times in the culture medium obtaining concentrations as indicated. Vehicle control condition is $0.25 \%$ ethanol. Auxin or vehicle control was included in the culture medium at the start of the assay or was pipetted into single wells during the assay at time points indicated. Plates were sealed with Breathe Easier sealing membrane (Diversified Biotech, BERM-2000). Luminescence was measured using a Luminometer (Berthold Technologies, Centro XS3 LB 960) for 0.5 seconds every 10 minutes for 72 hours at $20^{\circ} \mathrm{C}$ in a temperature-controlled incubator.

Luminescence data was analyzed using an automated algorithm for molt detection in MATLAB, with the option to manually annotate molts in a Graphical User Interface. In short, the hatch was detected by the first data point that exceeds the mean $+5^{*}$ stdev of the raw luminescence of the first 20 time points and also exceeds the raw luminescence by 3 . The molts were detected according to the method previously described (Olmedo et al., 2015) implemented in MATLAB.

\section{Hoechst 33258 staining}

Synchronized L1 worms by egg prep were plated and grown up to the L4 stage at $25^{\circ} \mathrm{C}$. Worms were then washed 3 times in $10 \mathrm{ml}$ of M9 buffer. After washing, Hoechst 33258 was added to $10 \mathrm{ml}$ to a final concentration of $1 \mu \mathrm{g} / \mathrm{ml}$ and incubated for $15 \mathrm{~min}$ on a rotating wheel. Incubation with Hoechst 33258 was then followed by 3 washes in M9 after which worms were concentrated in $1 \mathrm{ml}$ of M9 of which a few $\mu 1$ were mounted on a $2 \%(\mathrm{w} / \mathrm{v})$ agarose slide with $3 \mu$ l of levamisole $(10 \mathrm{mM})$ before a z-stack (z-stack interval 0.7$)$ was acquired using a LSM700 confocal microscope (Axio Imager Z2 (upright microscope) + LSM 700 scanning head, 40x/1.3 oil immersion objective, 6\% laser power, $378 \mathrm{~ms}$ exposure time, 512x300 pixels). The z-stack was mean projected and grey values were adjusted the 
same intensity range for all images (0-8000) in Fiji. Staining was quantified manually by assigning worms as being stained if blue signal in the nuclei was obvious.

\section{Western Blots}

Worms were synchronized by egg prep and grown on NG2\% plates at $25^{\circ} \mathrm{C}$ (Fig 4D) or in liquid culture with $\mathrm{OP} 50\left(\mathrm{OD}_{600} \sim 3.5\right)$ at $20^{\circ} \mathrm{C}$ with a concentration of 1 worm/ $\mu 1$ to the indicated developmental time points and $10^{\prime} 000-12^{\prime} 000$ worms per time point were harvested by pipetting the required amount of liquid to a $15 \mathrm{ml}$ falcon tube (Fig 4E). The worm samples were washed three times before the worm pellet was snap frozen in liquid nitrogen. $2 \mathrm{x}$ volume lysis buffer was added to $1 \mathrm{x}$ volume of frozen pellet and incubated at $95^{\circ} \mathrm{C}$ for 5 minutes. Samples were placed back on ice for 1 min and sonicated subsequently (Bioruptor, Diagnode, 13 cycles, $30 \mathrm{sec}$ on/off at $4^{\circ} \mathrm{C}$ ). The sonicated samples were centrifuged at $12,000 \mathrm{rcf}$ for $10 \mathrm{~min}$ at $4^{\circ} \mathrm{C}$ and the supernatant transferred to a new Eppendorf tube. Protein concentration in the supernatant was determined by Nanodrop (Protein function A280). Samples were diluted to $5 \mu \mathrm{g} / \mu 1$ final concentration with lysis buffer and stored at $-20^{\circ} \mathrm{C}$ or used for Western Blotting. For Western Blotting, $1 \mu 1$ of Bromophenol blue was added before incubation at $95^{\circ} \mathrm{C}$ for 3 minutes. $25 \mu 1$ of $5 \mu \mathrm{g} / \mu \mathrm{l}$ were loaded and the Western was run in MOPS buffer at 95V. The protein was transferred to a PVDF membrane for $1 \mathrm{~h}$ at $20 \mathrm{~V}$, followed by blocking in 5\% Skim Milk in TBS-T $(0.05 \%)$ for 1 hour. For 3xflag::BLMP-1 and Actin detection, the membrane was cut and incubated with primary antibodies for 1 hour at room temperature respectively. After primary antibody incubation, the membrane was washed $3 \mathrm{x} 15 \mathrm{~min}$ in TBS-T $(0.05 \%)$. In the case of actin, secondary antibody incubation for $45 \mathrm{~min}$ - 1 hour was performed and followed by $3 \mathrm{x} 15 \mathrm{~min}$ washes in TBS-T (0.01\%). Detection was performed using ECL reagents (RPN2232 and RPN2209, GE Healthcare) which was added to the membrane for 1 min before the signal was detected using the ImageQuant ${ }^{\mathrm{TM}}$ LAS 4000 (GE Healthcare). Antibodies and dilutions used: anti-FLAG- 
HRP (Sigma Aldrich A8592, 1:1’000), mouse anti-actin clone 4 (EMD Millipore MAB1501, 1:5’000), anti-mouse IgG HRP linked (GE Healthcare NXA931, 1:7’500).

\section{blmp-1(tm548) mutant time course}

Synchronized L1s by egg prep and overnight incubation in M9 were plated on food and grown at $25^{\circ} \mathrm{C}$. Samples were taken hourly from 1 hour until 24 hours of development and RNA isolation was performed using conventional RNA isolation with phenol chloroform extraction (adapted from Bethke et al. 2009). Sequencing libraries were prepared using the TruSeq Illumina mRNA-seq (stranded - high input) protocol followed by sequencing using the HiSeq 50 Cycle Single end reads protocol on HiSeq 2500.

\section{Processing of RNA sequencing results}

RNA-seq data were mapped to the $C$. elegans ce10 genome using STAR (Dobin et al., 2013) with default parameters (version 2.7.0f) and reads were counted using htseq-count (Anders et al., 2015) (version $=0.11 .2$ ).

Counts were scaled by total mapped library size for each sample. A pseudocount of 8 was added and counts were $\log _{2}$-transformed and then quantile normalized using normalize.quantiles() from the preprocess Core (version 1.52.1) (Bolstad, 2001) in R.

\section{Realignment of blmp-1 mutant RNA sequencing time courses}

In order to compare equivalent developmental time points, we had to realign the wild-type to the blmp- 1 mutant time course samples to account for differences in developmental tempo (Fig. S6). We realigned the time courses using the $\log _{2}$-transformed gene expression of all oscillating genes $(n=3$ '739). The mean normalized, 10 times oversampled and zero padded time series of each gene of the faster time course(s) were interpolated using a Lanczos kernel $(a=2)$ : 
kernel $=\frac{\left.2 * \sin (\pi * \mathrm{x}) * \sin \left(\pi * \frac{x}{2}\right)\right)}{\pi^{2} * \mathrm{x}^{2}}$

We then calculated the errors of the interpolated time points with the non-interpolated data of the slowest time course in $\log _{2}$ (Fig S6E), in our case the blmp-1(tm548) mutant samples of replicate 2. For each time point of the slowest time course, we selected the interpolated gene expression values of the time point with the smallest sum of the squared errors in both wildtype replicates and the blmp-1(tm548) replicate 1 to create a new realigned time course with the same number of time points as in the slowest condition (24 time points). Since the rhythmic process of oscillations leads to repetitive small errors, we had to limit the scanning for smallest error to the diagonal (Fig S6E).

In order to identify differentially expressed genes, we used edgeR (McCarthy et al., 2012; Robinson et al., 2009) on the realigned data. We first created counts from the $\log _{2}$-transformed realigned data again and ensured only non-negative counts by using $\operatorname{pmax}\left(2^{\wedge}(\right.$ realigned_log 2 data $\left.)-8,0\right)$ together with the real count data from the blmp-1(tm548) replicate 2 . We kept expressed genes by selecting genes which had at least 3 time points with counts higher than 5. These count data served as an input for edgeR (version 3.32.1). We then used the design matrix model.matrix $(\sim$ time + batch + time:treat $)$ to estimate the dispersion, run a QL fit and performed an F-test using glmQLFTest(fit, coef = coefficients_for_time_batch_time:treat). Since we tested many time points, the obtained pvalues were generally small and we would be able to obtain even small changes between samples by choosing a conventional p-value cutoff of 0.05 . Based on this circumstance, we selected genes not only based on a p-value but additionally also based on the mean of the absolute log-fold changes per gene to identify significant differentially expressed genes (Fig S7 A), resulting in our differentially expressed list of 1'655 genes (Fig 5A). The p-value 
threshold for identifying genes was set at: $-\log _{10}(p$-value $)=25^{*} 8^{\wedge}(-$ mean $($ abs $($ fold_changes $))$ +5 .

We ordered the gene expression and the fold changes of these 1'655 genes according to a 1dimensional tSNE performed on the fold changes of both replicates using the function Rtsne(fold_changes,dim $=1, p c a=F A L S E$,perplexity $=100$,thet $a=0.25$ ) from the Rtsne package (Krijthe, 2015) in R and used the first tSNE from the output for ordering (Fig 5A).

Amplitude and period calculation using Hilbert transform

Calculation of amplitudes and periods was performed as described in (Meeuse et al., 2020) in Python. The peak phases for Fig S6H were used from (Meeuse et al., 2020).

We analyzed the ModENCODE dataset (GSE25803) of BLMP-1 ChIPseq data to count the reads in promoter regions (defined as 1000bp around the ATG start codon, using WS220/ce10 annotations). We downloaded the raw fastq files (see supplementary table 1) and performed the alignment using the function qAlign(samples, genome = genomeName, pair $=' n o$ ', $c l O b j=c l$ ) from the QuasR package (Gaidatzis et al., 2015) in $\mathrm{R}$ to the C. elegans genome version ce10 since our RNA sequencing time course was also aligned to ce10 (see above). We used the resulting bam files as input for MACS2 (Zhang et al., 2008) using the command macs2 callpeak -t ChIP_samples -c control_samples-outdir output_directory -n ChIPvsCtrl_BLMP-1 -g ce-fBAM. To count the reads within the promoter regions we used the function $q$ Count(proj3, promoters_of_coding_transcripts, $c l O b j=c l$ ) from the QuasR package (Gaidatzis et al., 2015) in R and normalized the counts to the mean library size. To calculate the enrichment of counts in promoters between ChIP and control samples we added a pseudocount of $8, \log _{2}$ transformed the counts and calculated the enrichment using log2(promoter_counts_ChIP+8) - log2(promoter_counts_control+8). The mean enrichment 
of the two replicates per gene was used to threshold the genes of interest at a mean enrichment of 2-fold $\left(\log _{2}\right.$ of 1$)$.

\section{Single worm imaging}

Sample preparation and analysis were performed as described in Meeuse et al., 2020.

For scaling the GFP intensities in Figure 5 and S7 we used python with the numpy package (version 1.16.4). For Fig 5D, we scaled all single GFP traces from hatch until molt 4 exit to 200 datapoints $(=100 \%)$ using the function $\operatorname{interp}()$ from the numpy package (version 1.16.4). We then plotted the scaled values using seaborn's function lineplot(), using the standard deviation as error ( $c i=$ "sd”). In Fig S7 F and G we scaled all individual GFP intensity traces to the absolute mean duration of mock and blmp-1 RNAi separately for each larval stage in hours.

\section{Transgenic CRISPR strains aid::blmp-1}

Endogenous tagging of blmp- 1 with the auxin inducible degron (aid) was performed by CRISPR/Cas9 using dpy-10(cn64) co-conversion (Arribere et al., 2014) . The sgRNA sequence: 5' gccgaagagaacggtgccgg 3' was cloned into Not1-digested pIK198 (Katic et al., 2015) by Gibson assembly using the hybridized sequence from

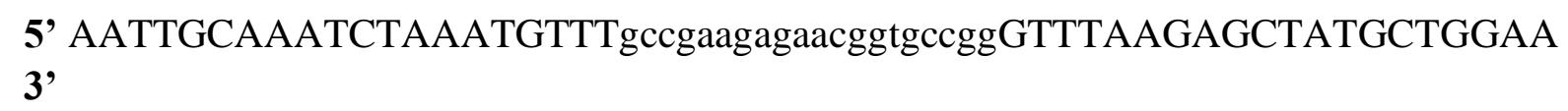
3'

and

5, TTCCAGCATAGCTCTTAAACccggcaccgttctcttcggcAAACATTTAGATTTGCAATT 3'.

The aid sequence was synthesized by IDT (Integrated DNA Technologies) as a gBlocks ${ }^{\circledR}$

Gene Fragments and contained 65 bp homology to blmp-1 locus, 30 bp downstream of the ATG startcodon. 
5'ttcgatctcattttaaacaaaacetgtaaaaaatgGGTCAAGGAAGTGGGGATGACGGTGTTCCGatgcc taaagatccagccaaacctccggccaaggcacaagttgtgggatggccaccggtgagatcataccggaagaacgtgatggtttcctg ccaaaaatcaagcggtggcceggaggcggcggcgttcgtgaagCCGGCACCGTTCTCTTCGGCTGCTGCG GCAGCTCACTCACCACCTCATTCTCCCCTTTCTGTCGG 3’.

The injection was performed in wild-type animals which were injected with $10 \mathrm{ng} / \mu 1 \mathrm{gBlock}$, $100 \mathrm{ng} / \mu \mathrm{l}$ sgRNA plasmid, $20 \mathrm{ng} / \mu \mathrm{l}$ AF-ZF-827 (Arribere et al., 2014), $50 \mathrm{ng} / \mu \mathrm{l} \mathrm{pIK155} \mathrm{(Katic}$ et al., 2015) and $100 \mathrm{ng} / \mu \mathrm{l}$ pIK208 (Katic et al., 2015).

\section{Transgenic CRISPR strain 3xflag::blmp-1}

N-terminal tagging endogenous $b l m p-1$ with $3 x F L A G$ was performed using the same $d p y$ -

10(cn64) co-conversion CRISPR/Cas9 strategy as used for the blmp-1::aid strain (see above, Arribere et al. 2014). The sgRNA with the sequence

\section{5' aaaaatgggtcaaggaagtg 3'}

was used to cleave genomic DNA followed by rescue with the $4 \mathrm{nM}$ Ultramer oligo sequence from IDT

5' $c t t c t c t t t c c t t c g a t c t c a t t t t a a a c a a a a c c t g t a a a a a+g$ GATTATAAAGACGATGACGATAAGCGTGAC TACAAGGACGACGACGACAAGCGTGATTACAAGGATGACGATGACAAGAGAGGAGC CGGATCTggtcaaggttcaggggatgacggtgttccgccggcaccgttctcttcggctgctgcggc 3'

Where bold underlined capital letters indicate the 3xFLAG with a spacer sequence in capital letters. The overlap to the genomic locus is indicated in small letters, comprising $47 \mathrm{bp}$ and $59 \mathrm{bp}$ overhang.

The injection was performed in wild-type animals which were injected with $20 \mathrm{ng} / \mu \mathrm{gBlock}$,

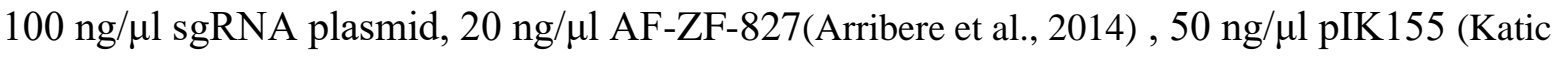
et al., 2015) and $100 \mathrm{ng} / \mu 1 \mathrm{pIK} 208$ (Katic et al., 2015). 
GFP reporters were cloned by Gibson assembly (Gibson et al., 2009) with amplified the promoters from genomic DNA using the primers listed below which were inserted into Nhe1digested pYPH0.14 as previously described, Meeuse et al., 2020. Transgenic animals were obtained by single copy-integration of the transgene into the ttTi5605 locus (MosSCI site) on chromosome II into EG6699 animals, with the published MosSCI protocol (Frøkjær-Jensen et al., 2012).

\section{Primers used to generate MosSCI plasmids:}

\begin{tabular}{|c|c|c|c|c|}
\hline Vector name & Backbone & Inserts & Primers & Primer sequence \\
\hline \multirow{2}{*}{ pYPH3 } & \multirow{2}{*}{ pYPH0.14 } & \multirow{2}{*}{$\begin{array}{l}\text { F58H1.2 } \\
\text { promoter }\end{array}$} & F58H1.2 promoter $\mathrm{FW} 1+\mathrm{OH}$ & $\begin{array}{l}\text { gcgtgtcaataatatcactcatagatgtatacta } \\
\text { atgaaggtaatagc }\end{array}$ \\
\hline & & & F58H1.2 promoter $\mathrm{RV} 1+\mathrm{OH}$ & $\begin{array}{l}\text { GCTAAGTCTAGACATcattcctgc } \\
\text { gtagaagcg }\end{array}$ \\
\hline \multirow{2}{*}{ pYPH4 } & \multirow{2}{*}{ pYPH0.14 } & \multirow{2}{*}{$\begin{array}{l}\text { R12E2.7 } \\
\text { promoter }\end{array}$} & R12E2.7 promoter $\mathrm{FW} 1+\mathrm{OH}$ & $\begin{array}{l}\text { gcgtgtcaataatatcactcaaattttaaaatat } \\
\text { ctttatttgaaaatt }\end{array}$ \\
\hline & & & $R 12 E 2.7$ promoter $\mathrm{RV} 1+\mathrm{OH}$ & $\begin{array}{l}\text { GCTAAGTCTAGACATcatgatgat } \\
\text { tgagatgtgttgaaa }\end{array}$ \\
\hline \multirow{2}{*}{ pYPH8 } & \multirow{2}{*}{ pYPH0.14 } & \multirow{2}{*}{$\begin{array}{l}\text { F33D4.6 } \\
\text { promoter }\end{array}$} & $F 33 D 4.6$ promoter $\mathrm{FW}+\mathrm{OH}$ & $\begin{array}{l}\text { gcgtgtcaataatatcactctgtgaaacggaaa } \\
\text { aaccatgc }\end{array}$ \\
\hline & & & F33D4.6 promoter $\mathrm{RV}+\mathrm{OH}$ & $\begin{array}{l}\text { GCTAAGTCTAGACATctgaaaca } \\
\text { tacatttaattctaattagt }\end{array}$ \\
\hline \multirow{2}{*}{ pYPH9 } & \multirow{2}{*}{ pYPH0.14 } & \multirow{2}{*}{$\begin{array}{l}\mathrm{C} 05 \mathrm{C} 10.3 \\
\text { promoter }\end{array}$} & C05C10.3 promoter $\mathrm{FW}+\mathrm{OH}$ & $\begin{array}{l}\text { gcgtgtcaataatatcactcaagttatcttttaaat } \\
\text { cttgaataaaaa }\end{array}$ \\
\hline & & & C05C10.3 promoter $\mathrm{RV}+\mathrm{OH}$ & $\begin{array}{l}\text { GCTAAGTCTAGACATtttatctgaa } \\
\text { tgaaaattttttaattt }\end{array}$ \\
\hline \multirow{2}{*}{ pYPH61 } & \multirow{2}{*}{ pYPH0.14 } & \multirow{2}{*}{$\begin{array}{l}\text { col-10 } \\
\text { promoter }\end{array}$} & $\begin{array}{l}\text { col-10 promoter } \mathrm{FW}+\mathrm{OH} \text { (to } \\
\text { pCFJ150) }\end{array}$ & $\begin{array}{l}\text { gggcgtgtcaataatatcactcatcttcttttcat } \\
\text { tttcaatct }\end{array}$ \\
\hline & & & 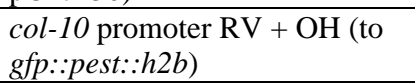 & $\begin{array}{l}\text { CCATGGCTAAGTCTAGACAT } \\
\text { gactgaaagccaggtac }\end{array}$ \\
\hline \multirow{2}{*}{ pYPH78 } & \multirow{2}{*}{ pYPH0.14 } & \multirow{2}{*}{$\begin{array}{l}F 16 B 4.4 \\
\text { promoter }\end{array}$} & $F 64 B 4.4$ promoter $\mathrm{FW}+\mathrm{OH}$ & $\begin{array}{l}\text { gcgtgtcaataatatcactcatctattatcgttaa } \\
\text { atgataactgtagt }\end{array}$ \\
\hline & & & $F 64 B 4.4$ promoter $\mathrm{RV}+\mathrm{OH}$ & $\begin{array}{l}\text { CCATGGCTAAGTCTAGACAT } \\
\text { gattgaacaaaatcggaatgatg }\end{array}$ \\
\hline
\end{tabular}

\section{Worm strains:}

HW1360: EG6699, xeSi131[F58H1.2p::GFP::H2B::Pest::unc-54 3', unc-119+] II

HW1361: EG6699, xeSi132[R12E2.7p::GFP::H2B::Pest::unc-54 3', unc-119+] II

HW1370: EG6699; xeSi136[F11E6.3p::GFP-H2B-Pest::unc-54 3’UTR; unc-119 +] II

(Meeuse et al., 2020)

HW1371: EG6699; xeSi137[F33D4.6p::GFP-H2B-Pest::unc-54 3'UTR; unc-119 +] II 
HW1372: EG6699; xeSi138[C05C10.3p::GFP-H2B-Pest::unc-54 3’UTR; unc-119 +] II HW1431: EG6699, xeSi160[daf-6ム4p::GFP::H2B::Pest::unc-54 3’UTR; unc-119 +] II HW1939: EG8079, xeSi296[eft-3p::luc::gfp::unc-54 3'UTR] II HW2521: EG6699,xeSi131[F58H1.2p::GFP::H2B::Pest::unc-54 3', unc-119+] II ;blmp-1 (tm548) (I)

HW2526: EG6699, xeSi440[dpy-9p::GFP::H2B::Pest::unc-54 3’UTR; unc-119 +] II (Meeuse et al., 2020)

HW2529: unc-119(ed3) III; ieSi59 [eft-3p::aid::GFP::unc-54 3'UTR + Cbr-unc-119(+)] III ; ieSi57 [eft-3p::TIR1::mRuby::unc-54 3'UTR, cb-unc-119(+)] II HW2532: EG8079, xeSi296[eft-3p::Luciferase::gfp::unc-54 3’UTR; unc-119 +] II ; blmp$1(\operatorname{tm} 548)(\mathrm{I})$

HW2533: EG6699; xeSi442[col-10p::GFP::H2B::Pest::unc-54 3’UTR; unc-119 +] II HW2639: blmp-1 (xe180[3xFlag::blmp-1]) I

HW2120: xe80 blmp-1(blmp-1::aid) I; EG8079, xeSi296 [eft-3p::luc::gfp::unc-54 3'UTR, unc-119(+)] II; EG8080, xeSi376 [eft-3p::TIR1::mRuby::unc-54 3'UTR, cb-unc-119(+)] III HW2033: bus-8 (e2885) X

HW2802: blmp-1 (xe180[3xFlag::blmp-1]) I ; xeSi296[eft-3p::Luciferase::gfp::unc-54 3’UTR; unc-119 +] II HW3028: EG6699,xeSi517[F16B4.4p::GFP::H2B::Pest::unc-54 3', unc-119+] II 


\section{Supplementary Figures}
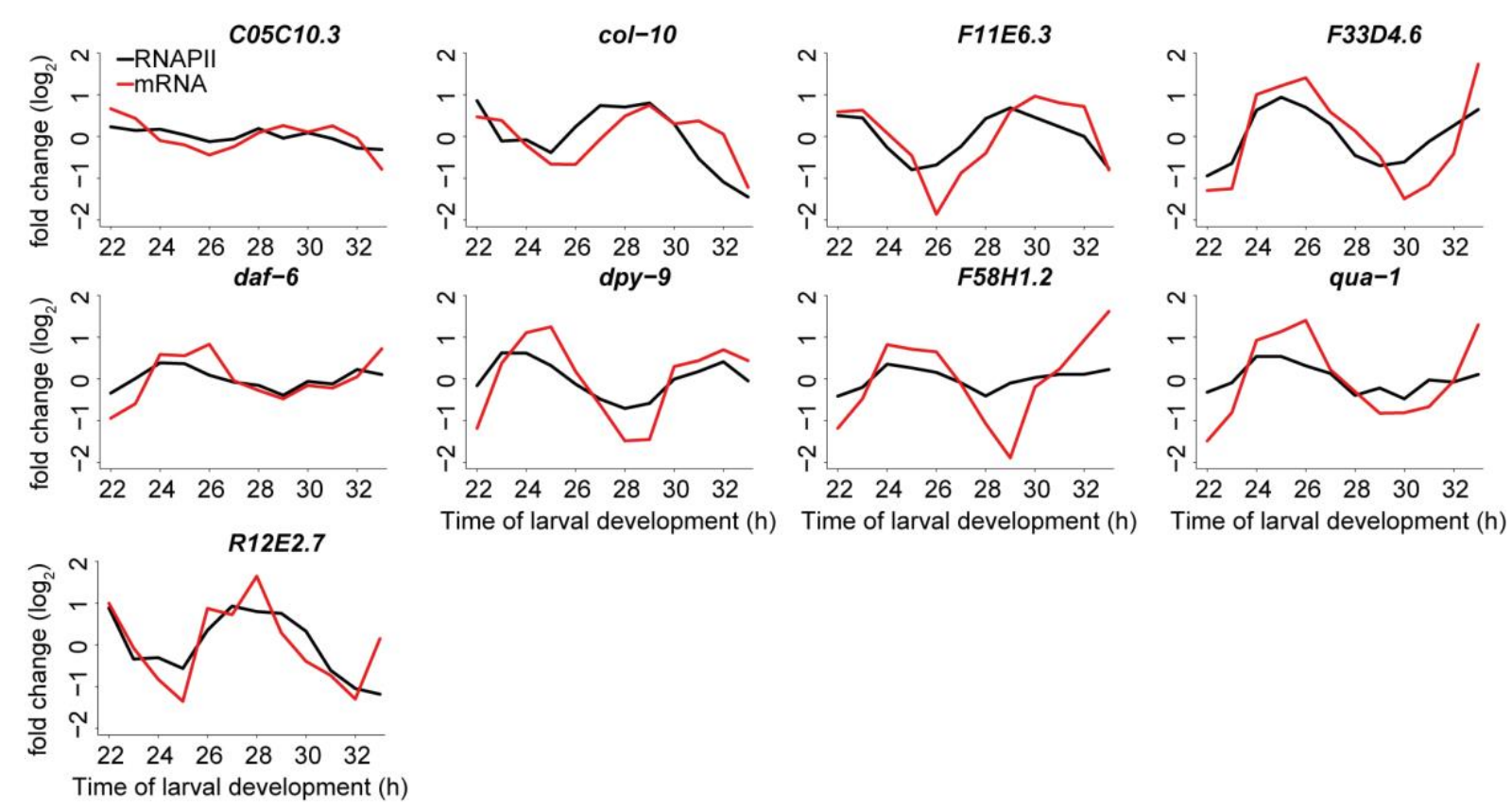

Time of larval development (h)

Fig S1: Comparison of RNAP II binding and mRNA level dynamics for specific genes,

\section{Related to Figure 1}

Comparison of RNAPII ChIPseq and RNA-seq patterns over time, for the same samples, for genes for which we also established promoter-based reporter transgenes. With the exception

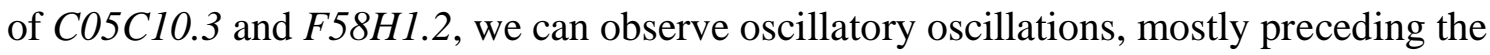
mRNA transcript oscillation. 
A
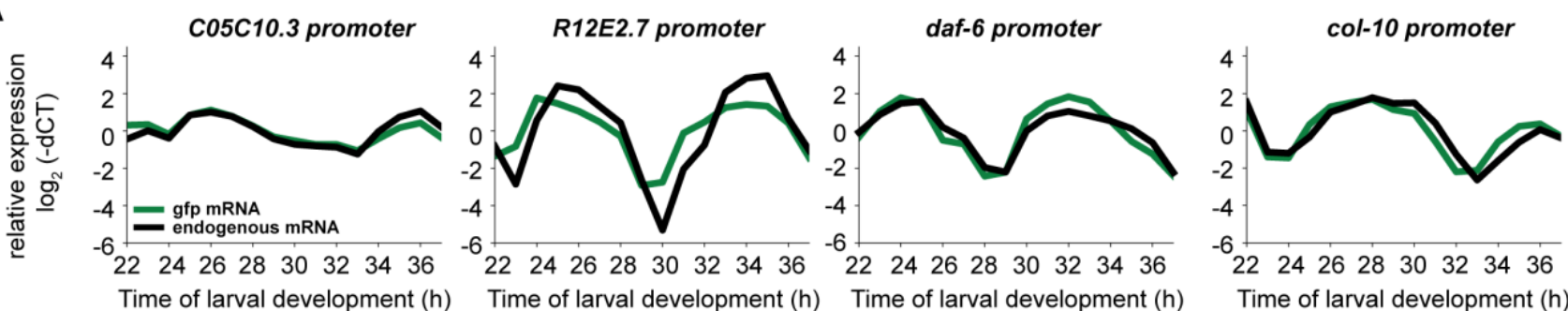

B
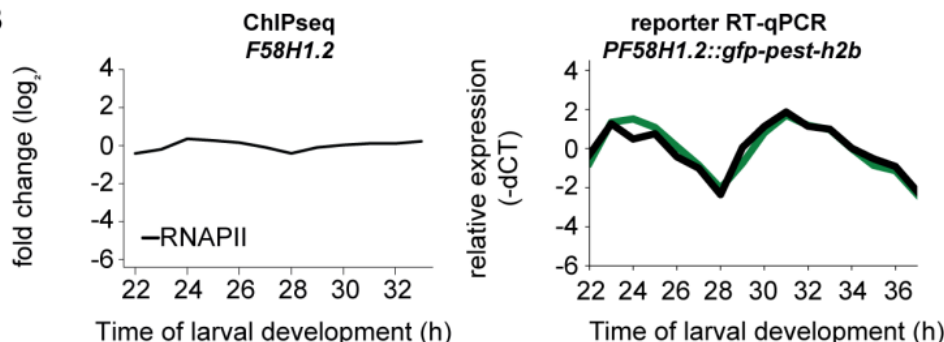

Fig S2: Additional transcriptional reporters investigated by RT-qPCR time courses;

\section{Related to Figure 1.}

A, Four additional transcriptional reporters were tested by RT-qPCR time courses. All except the reporter for $R 12 E 2.7$ recapitulated the amplitude and the peak phase. In the R12E2.7 case, we assume that we did not capture the entire promoter sequence or we that miss a distant regulatory element.

B, Comparison of ChIP-seq reads (left) and RT-qPCR reporter gfp and endogenous levels of F58H1.2. We detect a big amplitude in the RT-qPCR experiment even though the amplitude is low in the ChIP-seq experiment. 


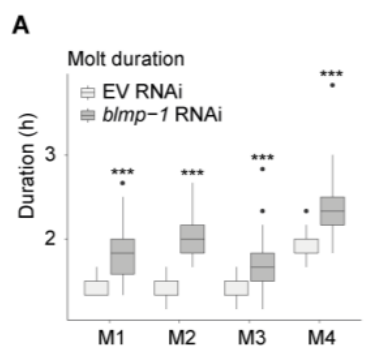

B $\quad$ C

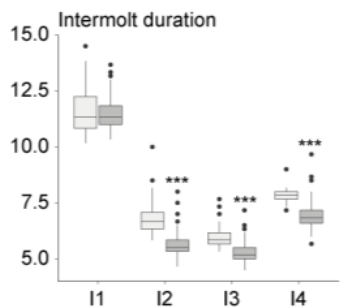

E

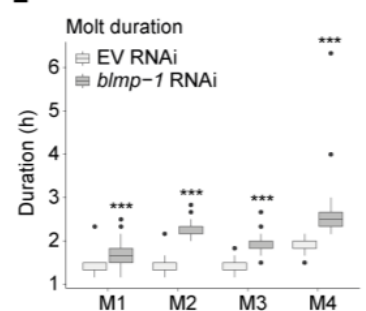

\section{$\mathbf{F}$}

Intermolt duration

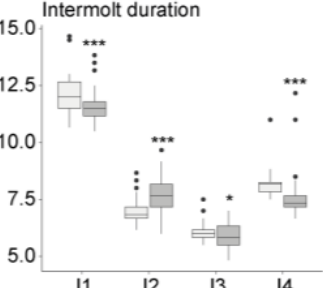

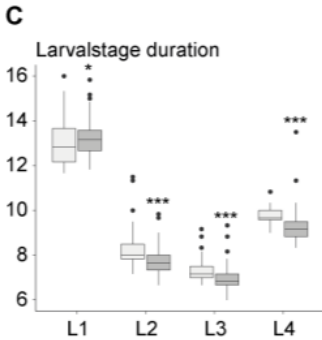

G

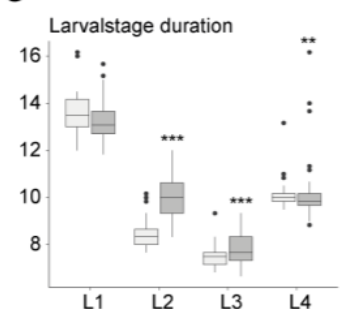

D

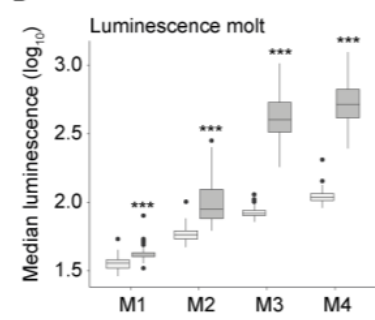

H

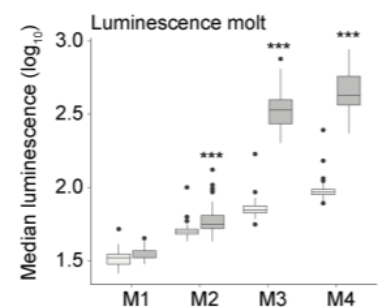

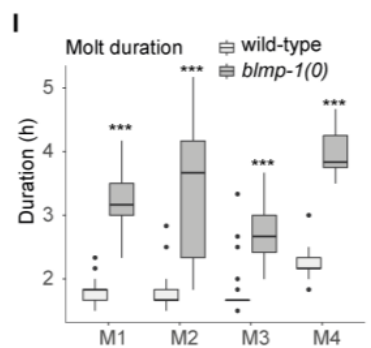
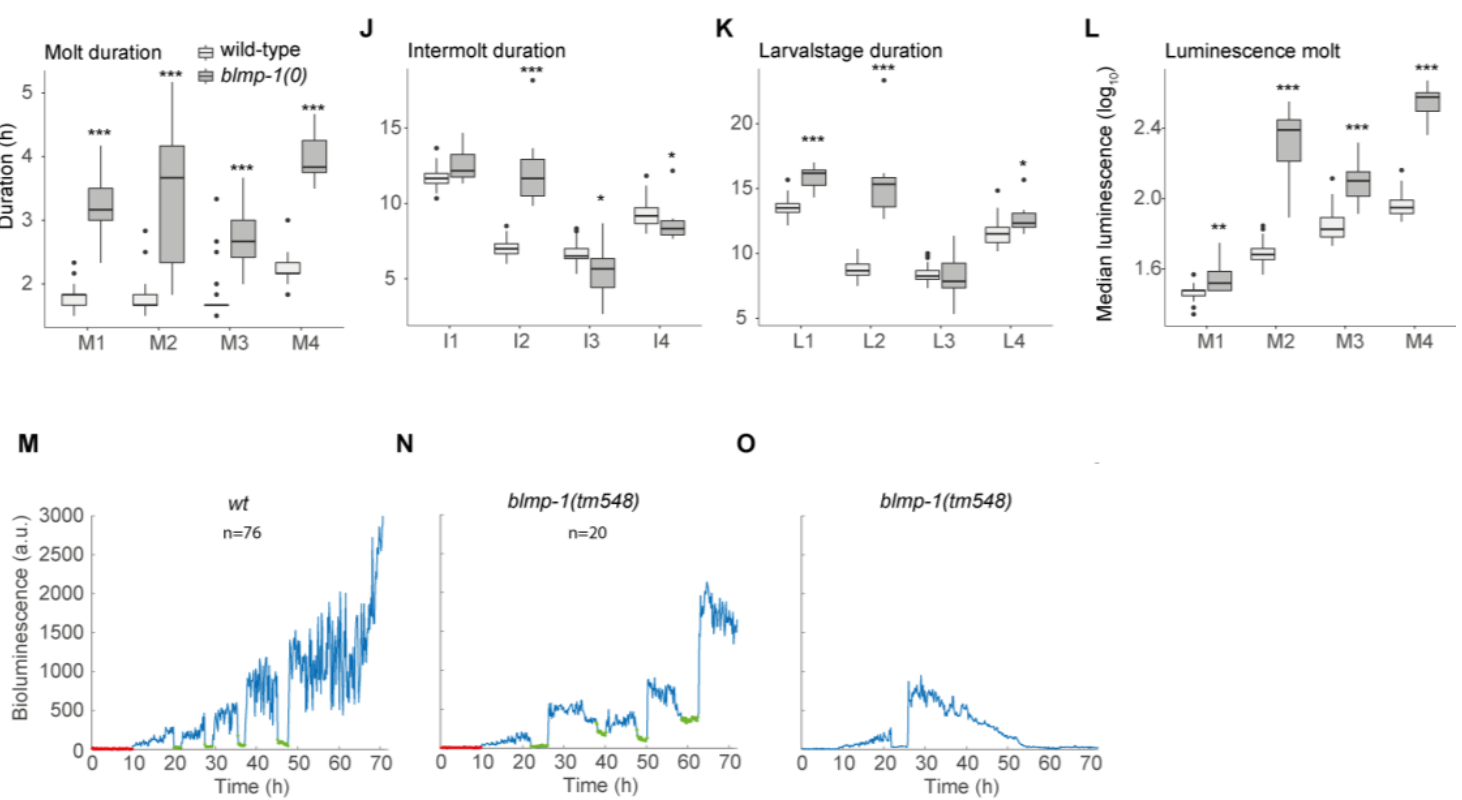

N
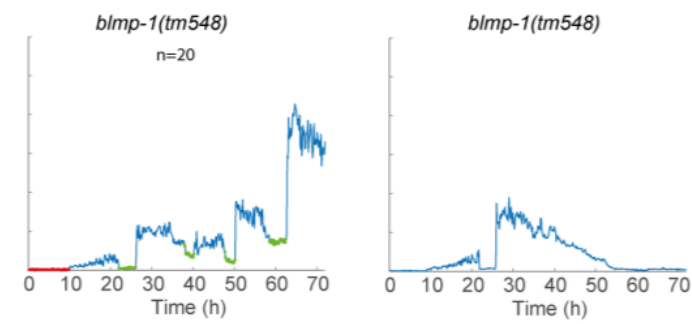

Fig S3: Replicates of experiments revealing developmental phenotypes upon BLMP-1

\section{loss, Related to Figure 2}

Boxplots of the quantification of single animal molt, intermolt and larval stage durations in mock and blmp-1 RNAi.

Both replicates confirm the longer molts $(\mathbf{A}, \mathbf{E})$, that are accompanied by shorter intermolts (B), or similar intermolt lengths (E, F), leading to similar $(\mathbf{C})$ or slightly longer $(\mathbf{G})$ larval stage durations in blmp- 1 RNAi compared to mock RNAi. Luminescence values are increased in both replicates during the molts $(\mathbf{D}, \mathbf{H})$. 
The second replicate of the blmp-1(0) mutant shows lengthening of molts (I) and similar or increased intermolt durations $(\mathrm{J})$ which leads to equal or increased larval stage durations $(\mathbf{K})$. Like in the blmp-1 RNAi condition, increased luminescence values are observed during the molt (L). Significantly different durations are indicated $(* \mathrm{P}<0.05$, ** $\mathrm{P}<0.01$, *** $\mathrm{P}<0.001$, Wilcoxon unpaired, two-sample test).

$\mathbf{M}-\mathbf{O}$, Representative luminescence traces from a wild-type (M, $\mathrm{n}=80)$ and surviving blmp1(tm548) mutant (N, n=7). Many blmp-1(tm548) mutants die (Fig. 2D) after exiting molts which is represented by a gradual decrease of luminescence after the exit from the first molt $(\mathrm{O})$. Boxplots represent the median as horizontal line and hinges extend to first and third quartiles, the whiskers extend up to $1.5^{*} \mathrm{IQR}$ (interquartile range). Data beyond that limit is plotted as dots. 
A
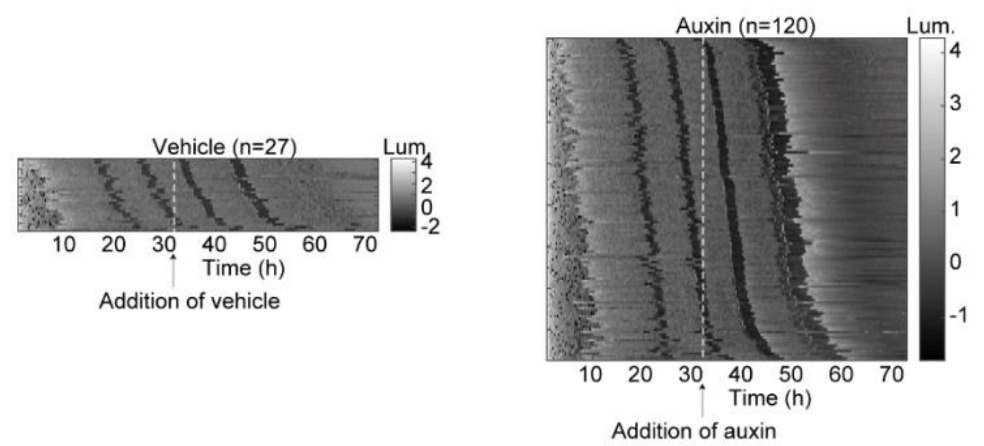

B
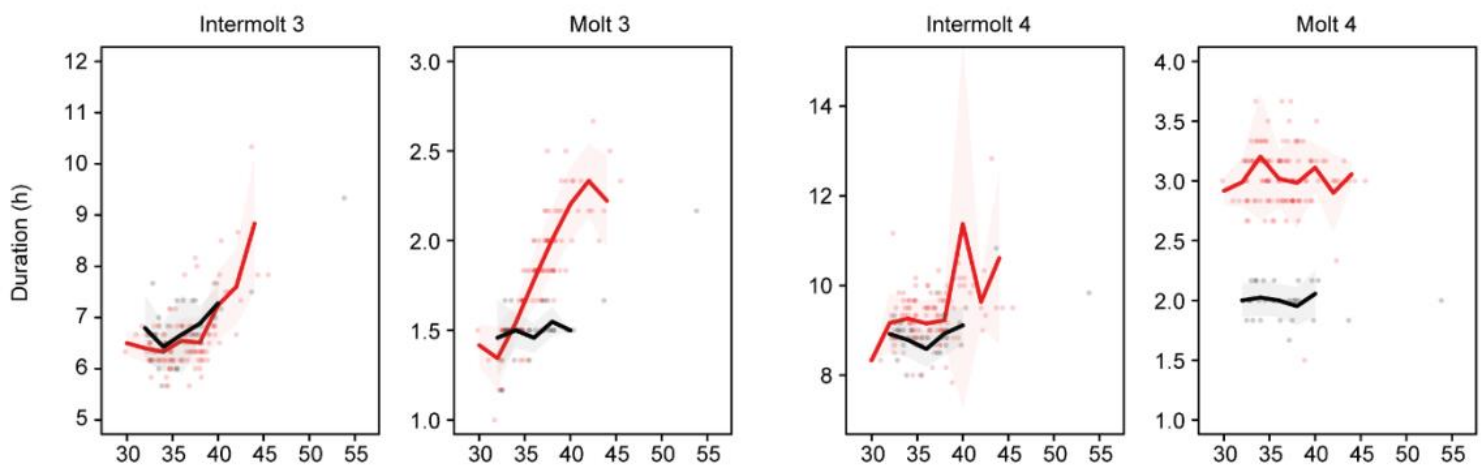

Fig S4: BLMP-1 depletion during L3 lengthens subsequent molts, Related to Figure 4.

Same experimental set-up as in Fig 4 with the exception that auxin was added at 32 hours after transferring single embryos into wells, when most animals are in the L3 stage.

A,B, Heatmaps showing trend-corrected luminescence (Lum.) traces; one animal per horizontal line. Vehicle $(0.25 \%$ ethanol; left) or $250 \mu \mathrm{M}$ auxin (right) were added at $\mathrm{t}=32 \mathrm{~h}$. $\mathrm{t}=0 \mathrm{~h}$ corresponds to the start of the assay. Embryos hatch at different time points and traces are sorted by entry into second molt. Dark grey indicates low luminescence and corresponds to the molts.

B, Duration of intermolt 3, molt 3, intermolt 4 and molt 4 plotted over time of molt 3 relative to auxin treatment in control and auxin treated animals shown in A. The line represents the mean and shading indicates the standard deviation. 


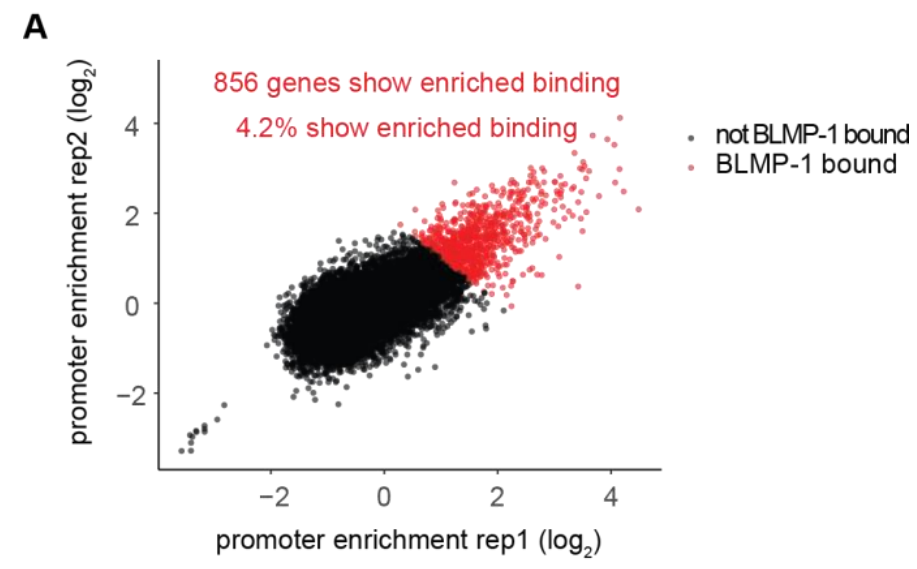

Figure S5: BLMP-1 binding is enriched on oscillating genes with a particular peak phase, Related to Figure 6

A, $\log _{2}$-fold enrichment of BLMP-1 binding to promoters (1000bp around the ATG start codon) for all coding genes $(n=20,392)$. Identified genes that show enriched binding (on average $\log _{2}>1$ across the replicates $)$ are labelled in red $(\mathrm{n}=856)$. 
bioRxiv preprint doi: https://doi.org/10.1101/2021.07.05.450828; this version posted July 5, 2021. The copyright holder for this preprint (which was not certified by peer review) is the author/funder, who has granted bioRxiv a license to display the preprint in perpetuity. It is made available under aCC-BY-NC 4.0 International license.

A

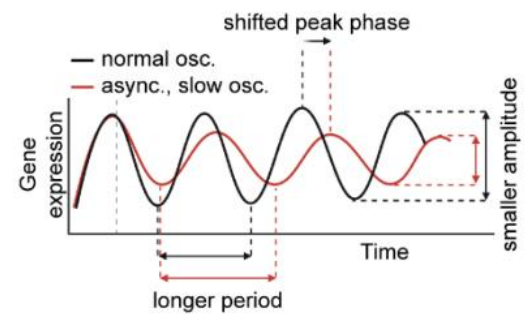

C

E
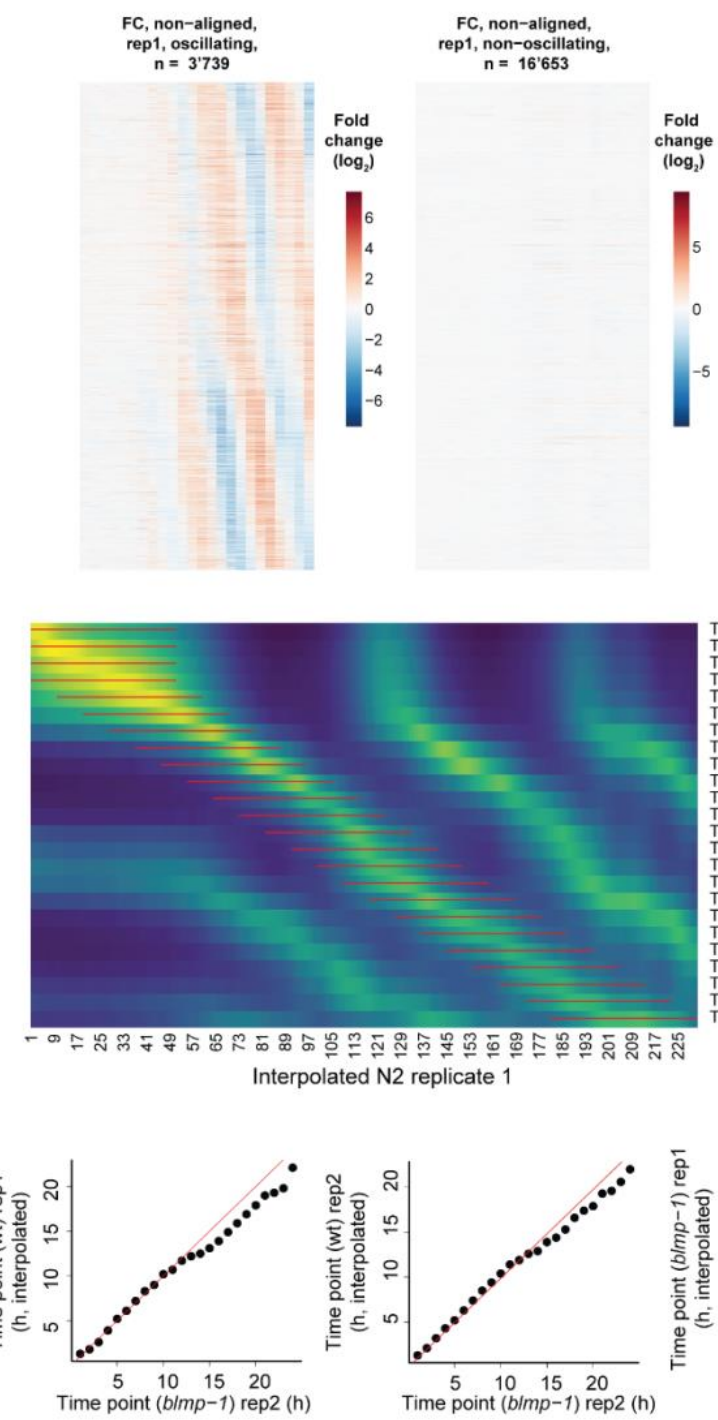

H

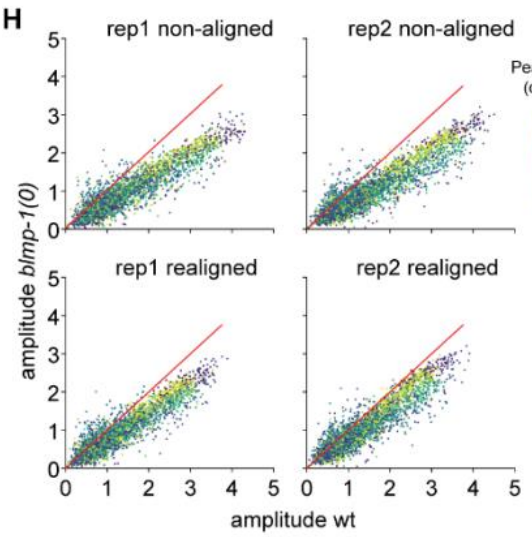

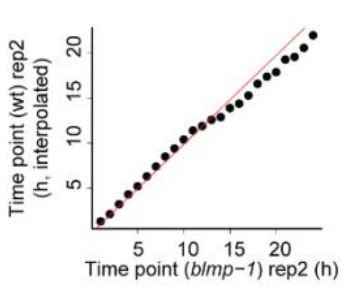

I
B

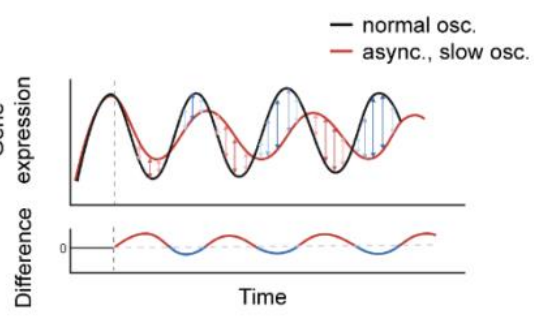

D
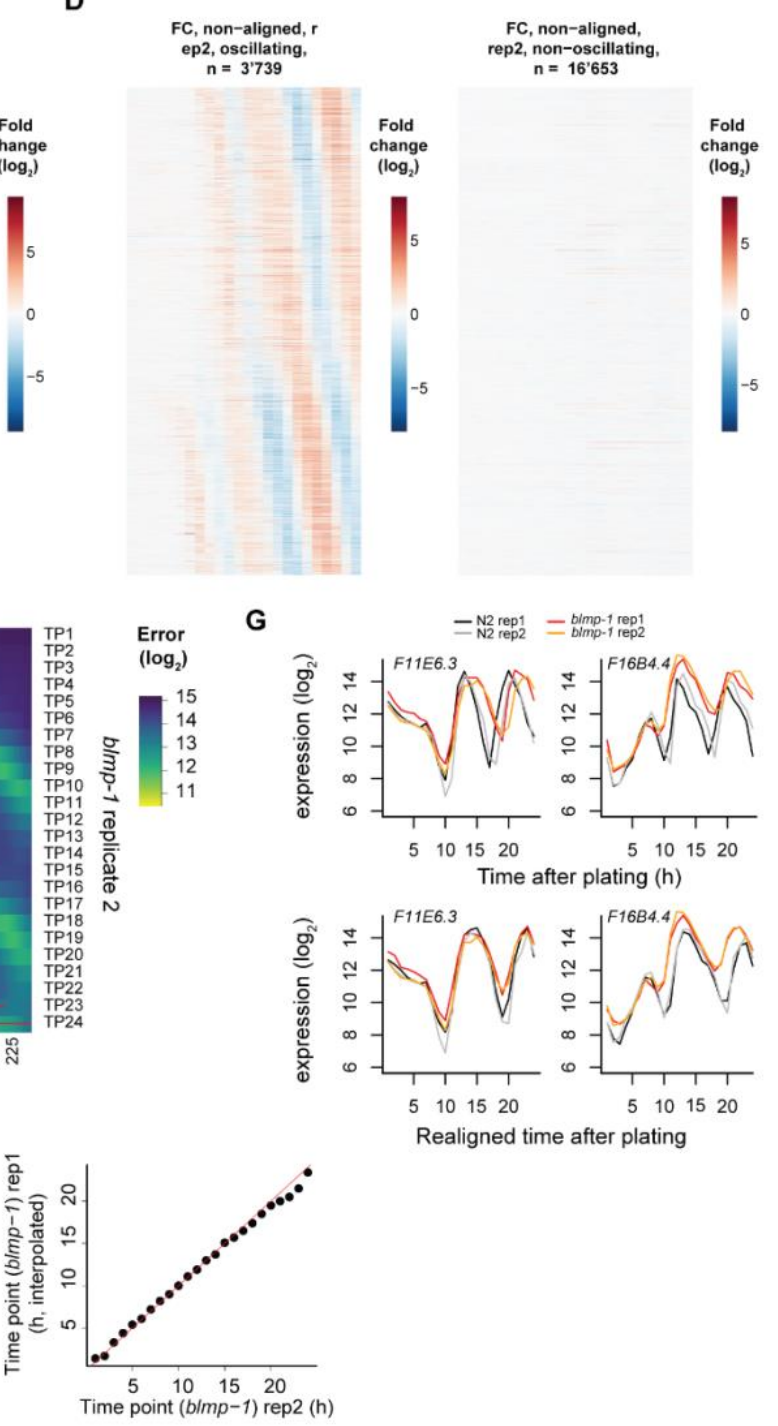

Realigned time after plating

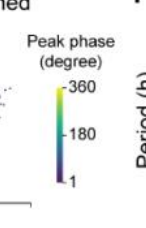

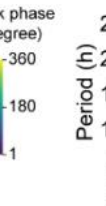
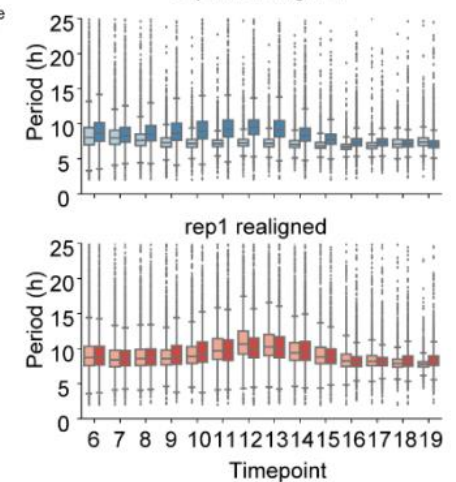

rep1 realigned rep1 non-aligned

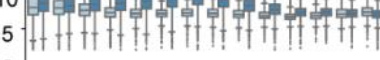

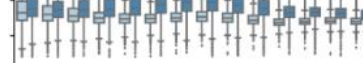

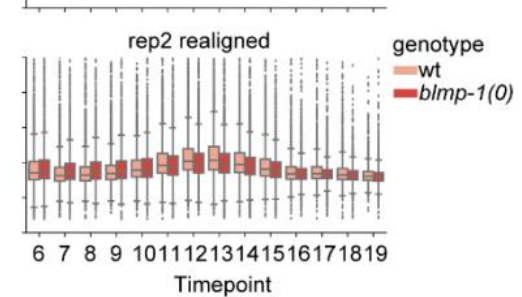

Fig S6: Temporal realignment corrects for different developmental tempos among 


\section{strains, Related to Figure 5}

A, Model of changes in oscillatory gene expression upon slower and unsynchronous development of a mutant (red) in comparison to a wild-type strain (black). In blmp-1 conditions, genes still coupled to development will slow down together with slower development. Furthermore, asynchronous development will result in reduced amplitudes in blmp-1 conditions. Created with Biorender.com

B, Resulting hypothetical rhythmic fold changes from a situation as in A.

C, D, Measured mean-centered fold changes of oscillating $(\mathrm{n}=3$ '739) and non-oscillating ( $\mathrm{n}$ $\left.=16^{\prime} 653\right)$ genes in $\log _{2}$ in two RNA sequencing experiments, comparing blmp-1(tm548) mutants with wild-type. Notice lack of overt differences in expression before $10 \mathrm{~h}$ of development and wide-spread and rhythmic fold changes in expression that become apparent thereafter, consistent with the altered developmental rates observed in Figure 2 \& S3.

E, After spline interpolation of the time courses, the sum of the squared error between the interpolated time course(s) and the blmp-1(tm548) mutant replicate 2 original $\log _{2-}$ transformed gene expression profile is calculated for all oscillating genes. For every time point of the blmp-1(tm548) replicate 2, the corresponding interpolated time point is chosen by identifying the smallest error. Since repetitive small errors occur due to the nature of the underlying oscillating gene expression, we limit the search to a diagonal sub-selection, indicated in red.

F, Calculation of the interpolated time point (h) that corresponds best to the blmp-1(tm548) replicate 2 timepoints (h).

G, $\log _{2}$-transformed gene expression of F11E6.3 and F16B4.4 before (top) and after (bottom) temporal realignment of the gene expression profiles of two replicates each of N2 wild type (black and gray traces) and blmp-1(tm548) mutant animals (orange).

25 H, Mean amplitudes calculated from the Hilbert transform of Butterworth-filtered signals (as in Meeuse et al., 2020) from time points 10 - 20 (to circumvent edge effects) of non-aligned and realigned $\log _{2}$-transformed expression data of oscillating genes. Color indicates the peak phase of the individual genes plotted.

I, Period over time calculated from the Hilbert transform of Butterworth-filtered signals (as in Meeuse et al., 2020) from time points 7 to 20 to circumvent edge effects. 
bioRxiv preprint doi: https://doi.org/10.1101/2021.07.05.450828; this version posted July 5, 2021. The copyright holder for this preprint (which was not certified by peer review) is the author/funder, who has granted bioRxiv a license to display the preprint in perpetuity. It is made available under aCC-BY-NC 4.0 International license.

A

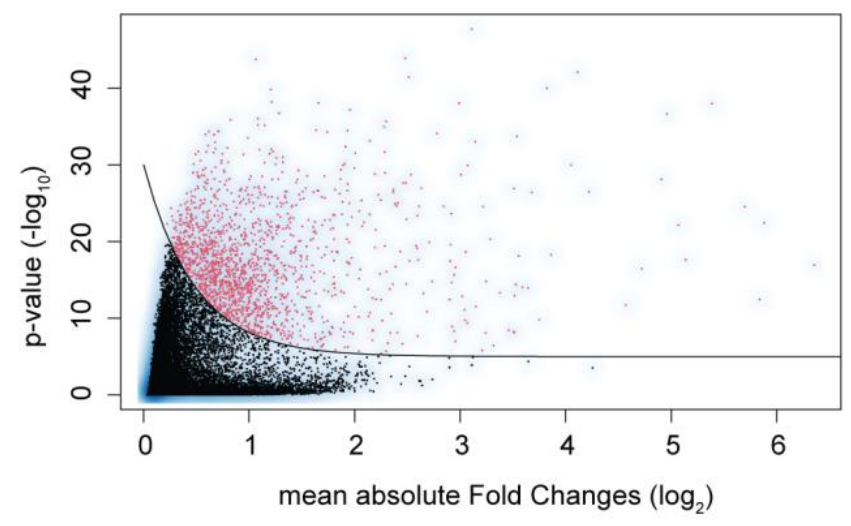

B

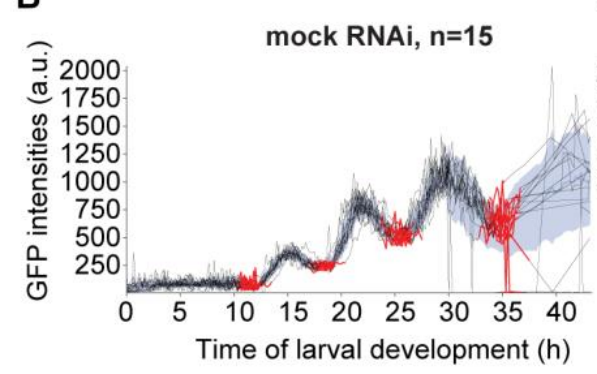

D

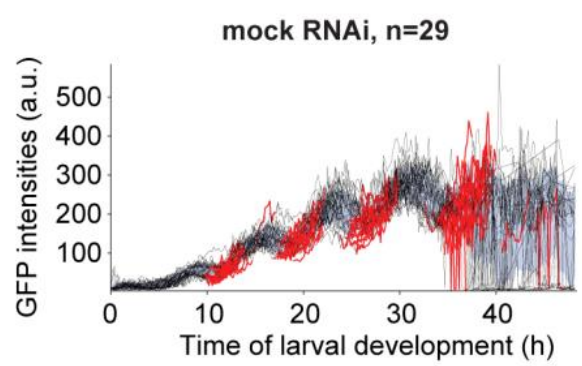

C

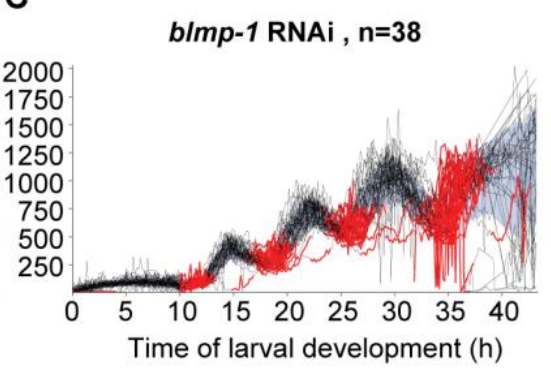

E

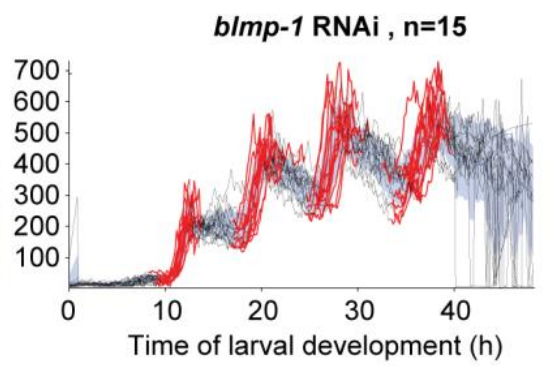

$\mathbf{F}$

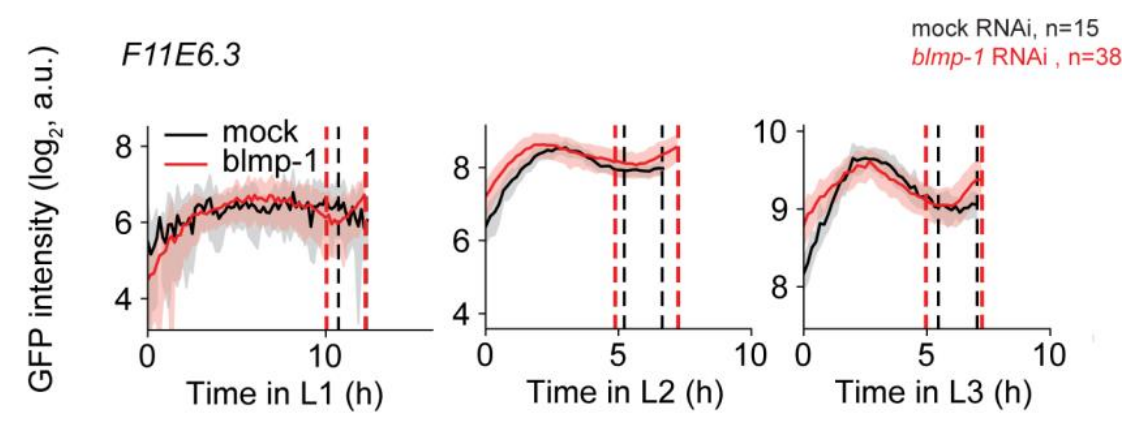

G

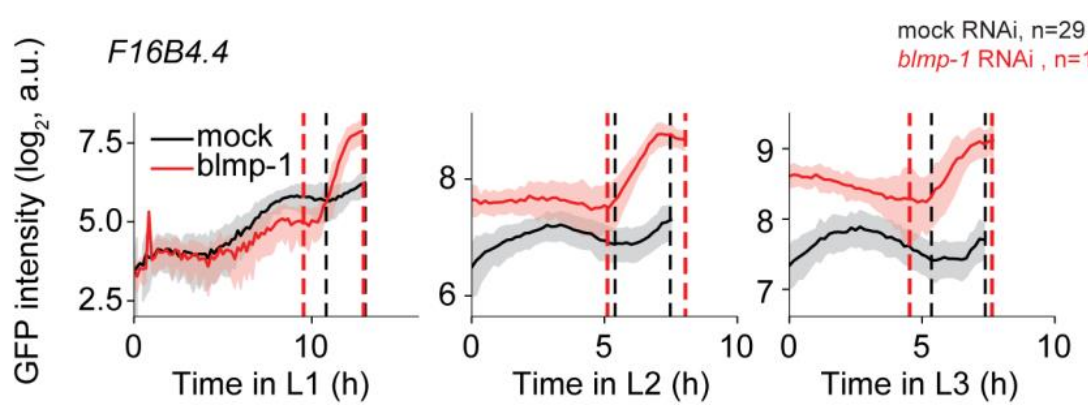


Figure S7: Identification of differentially expressed genes in blmp-1(0) mutant animals, Related to Figure 5

A, Scatter plot of - $\log _{10}$ p-values over mean of absolute fold changes between blmp-1(tm548) and $\mathrm{N} 2$ wild type samples over the course of development using the temporally realigned gene expression data from Figure S6. Dots represent individual genes, with genes above the indicated threshold line (Methods) colored in red.

B-E, Unscaled GFP intensities of single worm image analysis of F11E6.3p::gfp (B), (C) and F16B4.4p::gfp (D), (E) reporters in mock RNAs (B, D) or blmp-1 $(R N A i)$ treated animals. $\mathrm{t}=$ $0 \mathrm{~h}$ corresponds to hatch. Molting times are indicated in red.For missing time points we interpolated linearly, mostly after the fourth molt.

F, G, Per-larval-stage scaled $\log _{2}$-transformed mean GFP intensities of single worm image analysis from B-E. The GFP intensities were scaled to the mean larval stage of mock and blmp-1 RNAi separately for L1-L3 for the F11E6.3p::gfp (F) and the F16B4.4p::gfp (G) reporters. Vertical dashed lines indicate beginning and end of molt. The standard deviation is shown in shading. 
bioRxiv preprint doi: https://doi.org/10.1101/2021.07.05.450828; this version posted July 5, 2021. The copyright holder for this preprint (which was not certified by peer review) is the author/funder, who has granted bioRxiv a license to display the preprint in perpetuity. It is made available under aCC-BY-NC 4.0 International license.

A

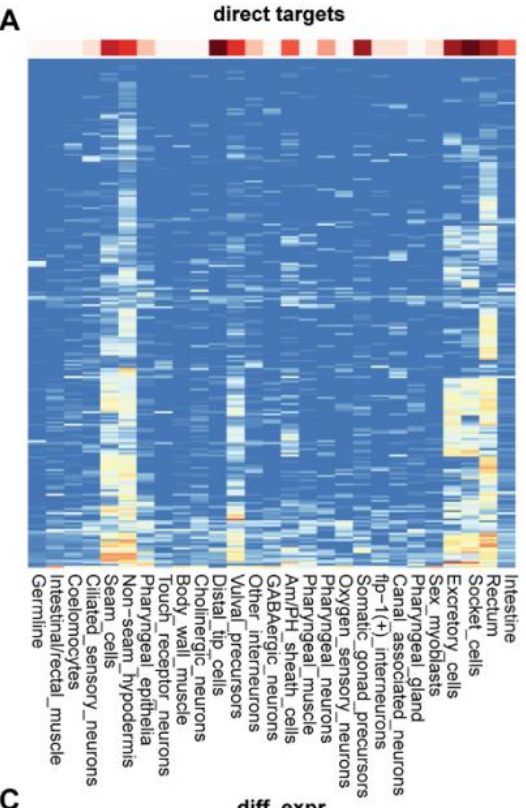

C
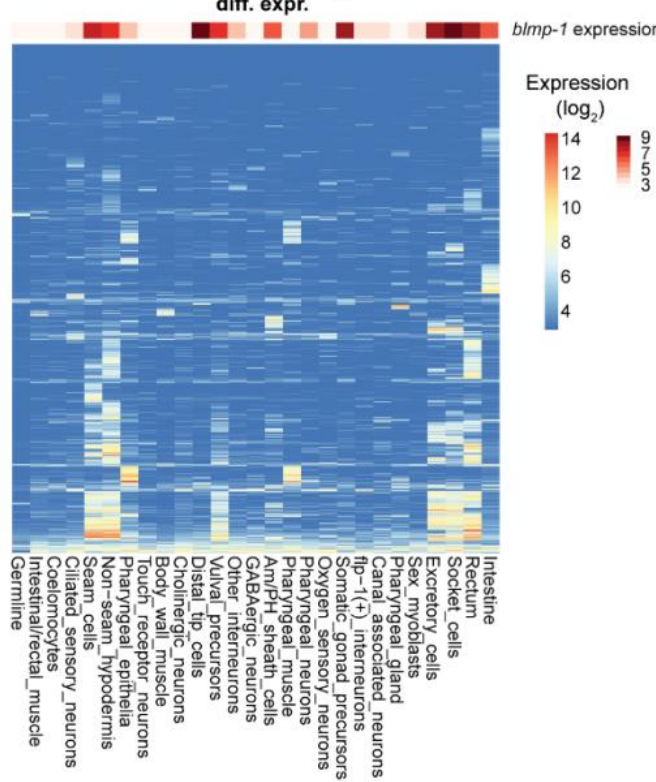

E

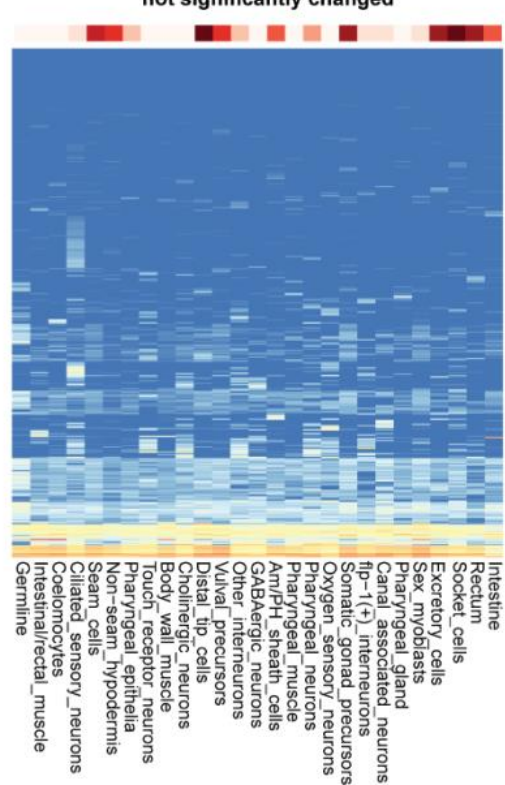

B direct targets

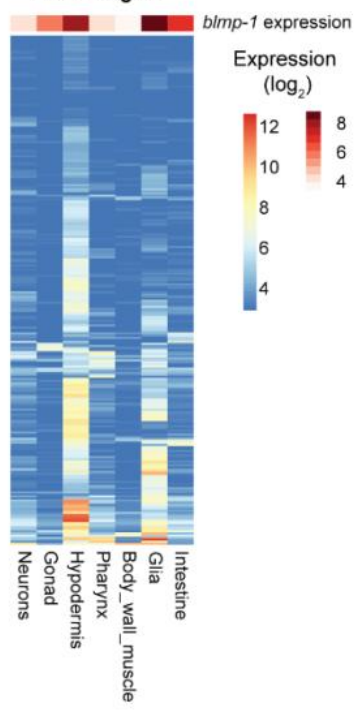

D

diff. expr.

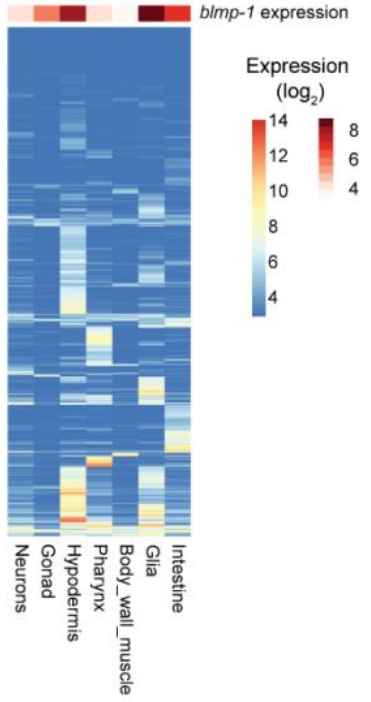

F not significantly changed

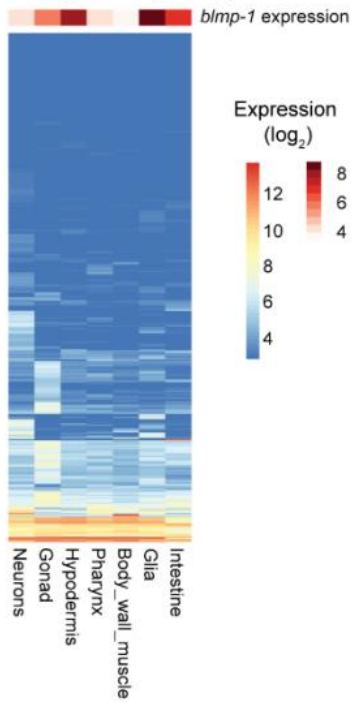




\section{Fig S8: Spatial expression patterns of different gene classes, Related to Figure 6}

Heatmaps are generated using single-cell RNA sequencing data from (Cao et al., 2017)

A, B, Expression of direct BLMP-1 target genes at the cellular (A) and tissue (B) level.

C, D, As in (A), (B) but for genes that are differentially expressed in blmp-1 mutant versus wild-type animals.

$\mathbf{E}, \mathbf{F}$, As in (A), (B) but for genes that are not differentially expressed in blmp- 1 mutant versus wild-type animals. 
bioRxiv preprint doi: https://doi.org/10.1101/2021.07.05.450828; this version posted July 5, 2021. The copyright holder for this preprint (which was not certified by peer review) is the author/funder, who has granted bioRxiv a license to display the preprint in perpetuity. It is made available under aCC-BY-NC 4.0 International license.

A

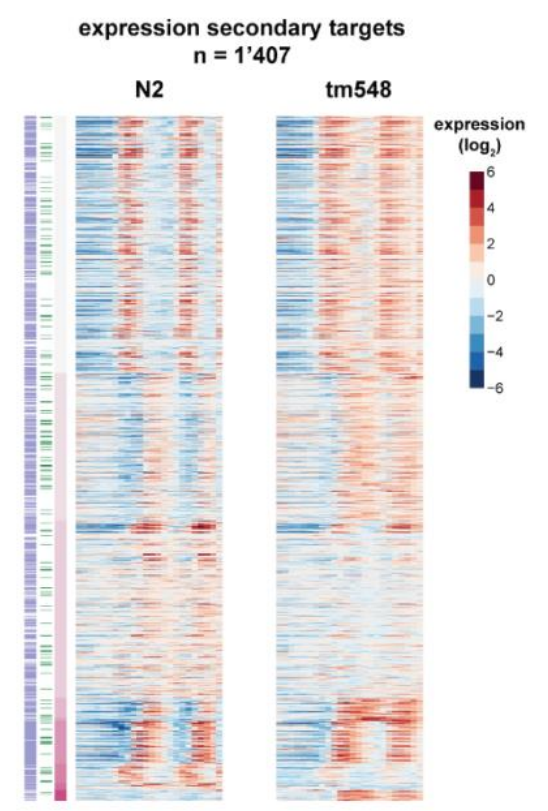

FC secondary targets $n=1 ' 407$

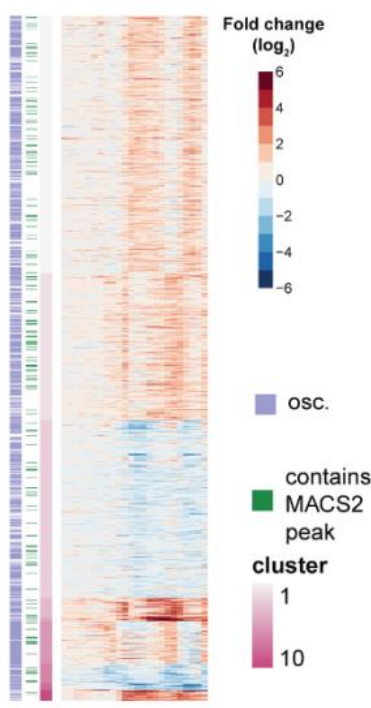

B

C
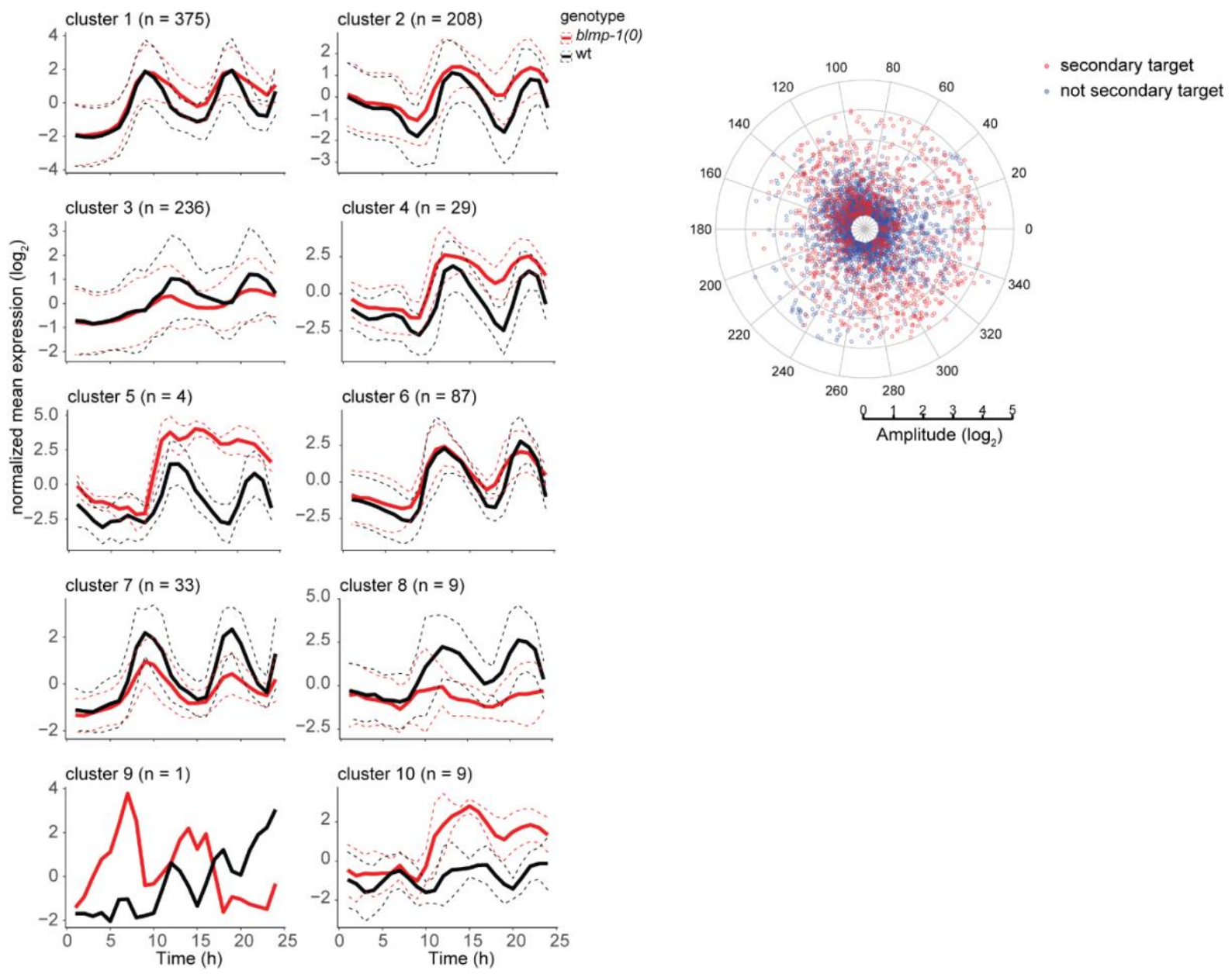

Fig S9: Secondary targets are affected in oscillatory gene expression but don't display phase specificity 
A, Heatmap of $\log _{2}$-transformed gene expression and fold changes of secondary BLMP-1 targets.

B, Mean-normalized mean expression and peak phase distributions of oscillating secondary target genes according to the 10 clusters in A. The dashed lines represent the standard deviation.

C, Peak phases (circular axis, degree) and amplitudes (radial axis, $\log _{2}$ ) of secondary target genes (red) and the remaining oscillating genes (blue). 
bioRxiv preprint doi: https://doi.org/10.1101/2021.07.05.450828; this version posted July 5, 2021. The copyright holder for this preprint (which was not certified by peer review) is the author/funder, who has granted bioRxiv a license to display the preprint in perpetuity. It is made available under aCC-BY-NC 4.0 International license.

A
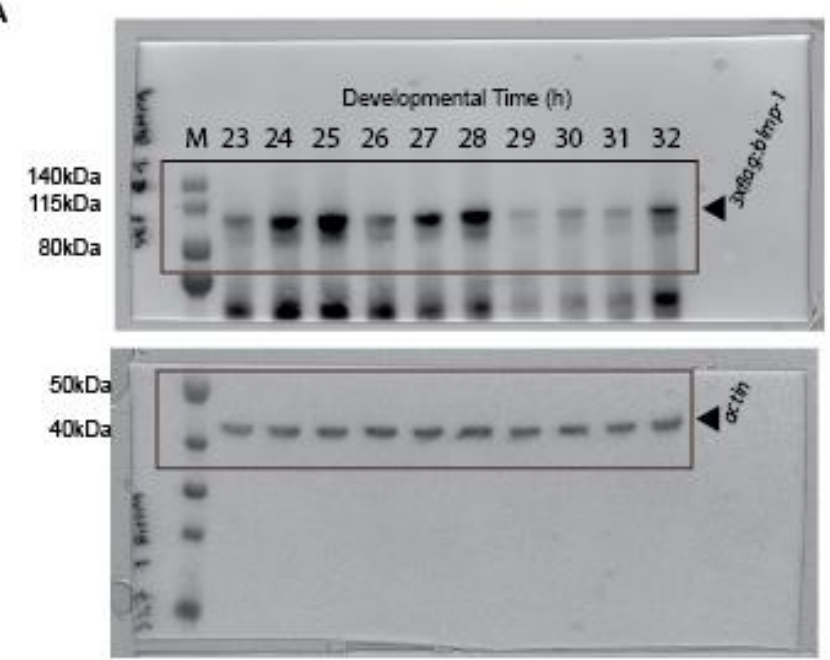

B
Developmental Time (h)
C

$\begin{array}{llllllllllllllllllllll}\text { M } & 17 & 18 & 19 & 20 & 21 & 22 & 23 & 24 & 25 & 28 & 27 & 28 & 29 & 30 & 31 & 32 & 33 & 34 & 35 & 36 & \text { M }\end{array}$

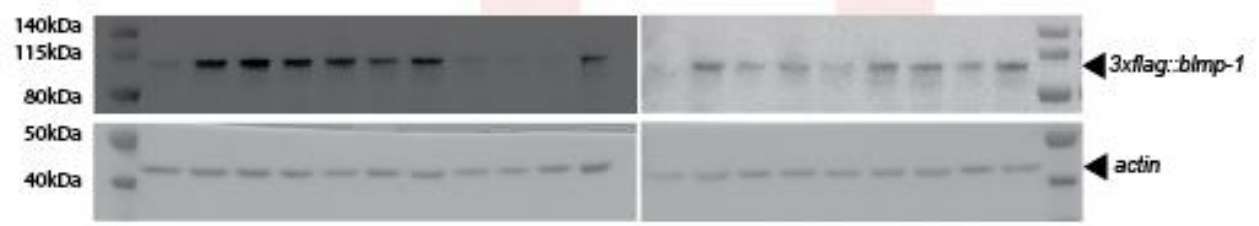

D

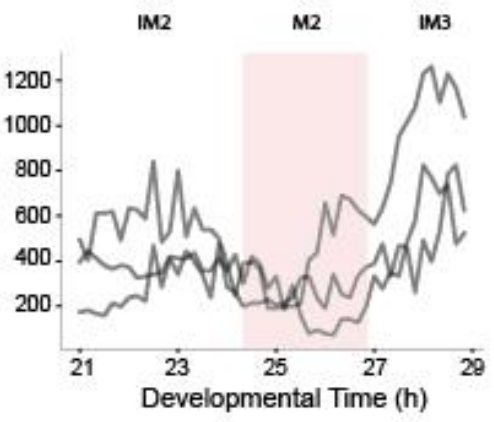

E IM3 M3 IM4 M4 adults

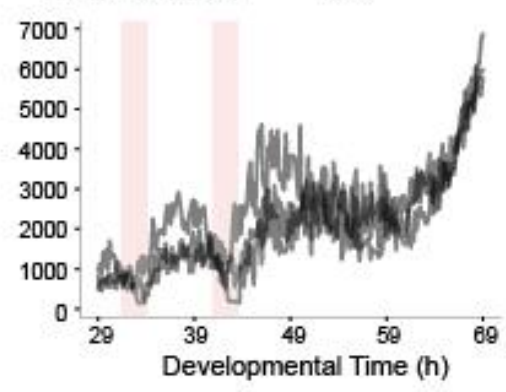

G
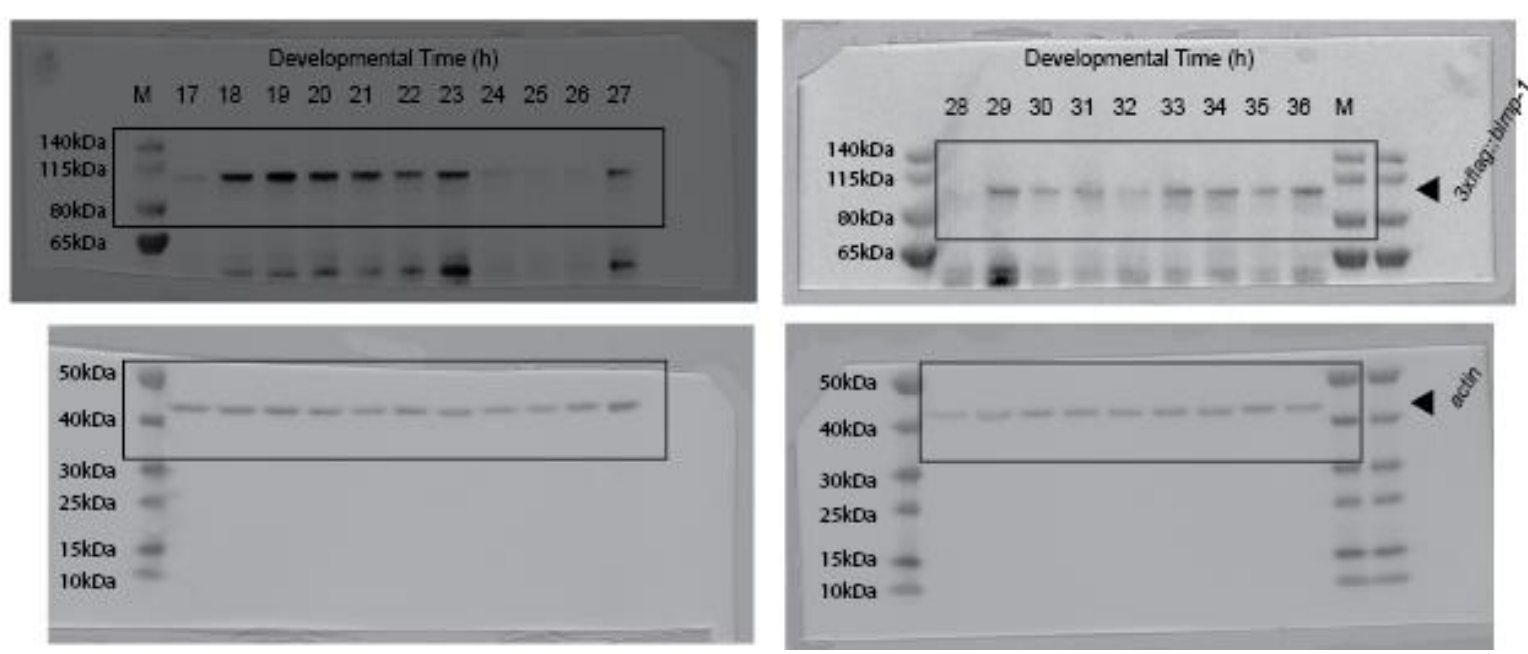

Fig S10: Western Blot of 3xflag::blmp-1 worms (HW2639 and HW2802) 
A, Uncropped Western Blot images of 3xFLAG::BLMP-1 protein in HW2639 worms grown on $\mathrm{NG} 2 \%$ plates at $25^{\circ} \mathrm{C}$.

B, C, 3xFLAG::BLMP-1 protein detected by Western Blot in HW2802 worms grown in liquid culture at $20^{\circ} \mathrm{C}$. Protein abundance from early L2 to early L3 stage (B) and early L3 to late L4 stage (C) originating from the same time course. The molts, as determined by luciferase assays $(\mathrm{D}, \mathrm{E})$, are indicated in salmon.

D, E, Luminescence intensities over time of multiple HW2802 worms ( $<10$ for each of the 3 wells on the plate), starting from 21 hours (D) or 29 hours (E) of development. The drop in signal from 25 to 26 hours (D) and 32 to 34 hours (E) is indicative for the molt. The molt assignment in this case was performed on multiple worms cultured in one well mainly to speed up handling time. However, thus the molt assignment is less accurate than in Fig S5 resulting from slight developmental asynchrony present in the population.

F, G, Uncropped images of Western Blots shown in B and C. 